\title{
Monoterpene responses to interacting effects of drought stress and infection by the fungus Heterobasidion parviporum in two clones of Norway spruce (Picea abies) ${ }^{\text {is }}$
}

\author{
Anat Madmony ${ }^{\mathrm{a}}$, Roberto Tognetti ${ }^{\mathrm{b}}$, Letizia Zamponi ${ }^{\mathrm{c}}$, Paolo Capretti ${ }^{\mathrm{c}}$, Marco Michelozzi ${ }^{\mathrm{d}, *}$ \\ a Department of Environmental Studies and Agriculture, Beit Berl College, Doar beit Berl, Israel \\ ${ }^{\mathbf{b}}$ Dipartimento di Agricoltura, Ambiente e Alimenti, Università degli Studi del Molise, Via Francesco De Sanctis, 86100 Campobasso, Italy \\ ${ }^{\mathrm{c}}$ Dipartimento di Scienze delle Produzioni Agroalimentari e dell'Ambiente (DISPAA), Università degli Studi di Firenze, P.le delle Cascine 28, 50144 Firenze, Italy \\ ${ }^{\mathrm{d}}$ Istituto di Bioscienze e Biorisorse - C.N.R., via Madonna del Piano 10, 50019 Sesto F.no, Firenze, Italy
}

\section{A R T I C L E I N F O}

\section{Keywords:}

Drought stress

Heterobasidion parviporum

Monoterpenes

Norway spruce

Resistance

\begin{abstract}
A B S T R A C T
To study simultaneous biotic and abiotic stress effects on monoterpene mediated defence response, four-year-old seedlings of two clones of Picea abies (c386: "relatively resistant" c386; c171: "relatively susceptible" clone 171) were inoculated with an isolate of rot fungus Heterobasidion parviporum and simultaneously subjected to a drought stress recovery cycle. Fungal growth into the wood was generally higher in well-watered (W) than in drought-stressed (D) plants. As predawn water potential decreased, gas exchange and maximal photochemical efficiency of PSII also declined. Water potential and chlorophyll fluorescence showed differences between clones in D plants, c386 being more tolerant than $\mathrm{c} 171$, while stomatal conductance and net photosynthesis differed between clones in W plants; c171 showing higher values than c386. A recovery of physiological functions was observed after re-watering. The clones showed different constitutive monoterpene profiles. The infection generally caused some extensive compositional changes in the relative contents of several monoterpenes and their enantiomers; also, sterile infection (wounded only samples) affected the relative proportions of monoterpenes, although to a lesser extent than reactions elicited by the fungus. The trend in monoterpene response to disease treatments was the same in D and W plants of both clones. In particular, higher proportion of $\delta$-3-carene was found constitutively in c386 in comparison with c171; besides, infection with $H$. parviporum increased the relative contents of $\delta$-3-carene in all the samples, even if proportions of this monoterpene in wounded plus inoculated tissues of c386 were significantly higher than cortical tissues from non-inoculated branches only in $\mathrm{W}$ seedlings 44 days after treatment.

Although drought stress decreased the total absolute contents of monoterpenes, total monoterpene concentrations significantly increased in response to infection by $H$. parviporum.
\end{abstract}

\section{Introduction}

Regional-scale vegetation mortality events appear to be increasing in a variety of biomes throughout Earth, and are frequently associated with increased temperatures, droughts, and often (but not always) with outbreaks of biotic agents, such as insects and pathogens (Allen et al., 2010; Das et al., 2016; Wong and Daniel, 2017). The physiological mechanisms underlying tree mortality involve the water and carbohydrates storage and transport systems (McDowell et al., 2011). In temperate zones, drought is associated with enhancement of disease development in several tree species by many pathogens that may affect both the tree crown and the root system (Madar et al., 1989). Indeed, the heat wave and the severe drought that Western Europe experienced in 2003 had serious consequences for forest health (Desprez-Loustau et al., 2006).

Based on predictions of future climate change, increased frequency and severity of drought in combination with other disturbances (including fungal diseases) are expected to affect the health of Norway spruce (Picea abies (L.) Karst.) (Hanewinkel et al., 2013), the most important commercial conifer in Europe. This forest species spreads from Siberia to the Northern and Central Europe extending the area of its natural range area to the Alps and Balkan Peninsula. In temperate European forests dominated by Norway spruce, recent wind storms and beetle outbreaks have caused widespread damage (Seidl et al., 2011).

\footnotetext{
This article is part of a special issue entitled "Experiments with trees: from seedlings to ecosystems" published at the journal Environmental and Experimental Botany 152C.

* Corresponding author.

E-mail addresses: Anat@Madmony.co.il (A. Madmony), tognetti@unimol.it (R. Tognetti), lae76@virgilio.it (L. Zamponi), paolo.capretti@unifi.it (P. Capretti), marco.michelozzi@cnr.it (M. Michelozzi).
} 
The distribution area of Norway spruce fits well with the natural range of the stem and root rot fungus Heterobasidion annosum sensu lato. This fungus is considered one of the most harmful pathogens for conifer trees in the Northern hemisphere causing remarkable losses of merchantable wood volumes both in plantations as well in natural forests (Woodward et al., 1998). Norway spruce stands affected by $H$. annosum are more susceptible to bark beetle and are more likely to suffer from windthrow (Korhonen and Stenlid, 1998).

Exposure to drought may also make trees more vulnerable to insects (Gaylord et al., 2013) and pathogens (Niinemets, 2010). Drought stress enhanced the development of Diplodia pinea on Italian cypress (Madar et al., 1995), Fusarium compactum on Cupressus sempervirens (Madar et al., 1996) and Sphaeropsis sapinea on Pinus resinosa (Blodgett et al., 1997a,b). A combination of mass inoculation (Leptographium wingfieldii) and drought stress resulted in a dramatic loss of stem hydraulic conductivity in Pinus sylvestris that was paralleled by conspicuous damage to the sapwood (Croisé et al., 2001). Water-stressed plants of Eucalyptus globulus were more susceptible to Neofusicoccum eucalyptorum than nonstressed ones, drought-primed plants being slightly less susceptible to the attack of this pathogenic fungus than the non-primed ones (Barradas et al., 2018). In addition to drought, other factors triggered by climate change may favour certain pathogens, i.e., warming temperature, pathogen migration and vegetation shift (La Porta et al., 2008).

The influence of drought on $H$. annosum, Phytophthora cinnamomi, and Armillaria spp. was outlined by several authors (Lindberg and Johansson, 1989; Brasier et al., 1993; Wargo and Harrington, 1991; Puddu et al., 2003). Inoculations experiments with seedlings of Norway spruce showed that drought may affect the rate of growth of $H$. parviporum inside the tree (Lindberg and Johansson, 1992). Biotic stress caused by pathogen infection triggers the expression of defence related genes and induces systemic host responses (Hammerschmidt, 1999). Gori et al. (2013) found that, H. parviporum is somewhat more aggressive in adult trees growing at low than high elevation, causing a more rapid decline of tree growth and decreasing the ability of host Norway spruce to cope with drought. Increasing drought has the potential to favour diseases caused by Heterobasidion spp., eventually leading to tree mortality (Linares et al., 2010).

In conifers, induced stress responses include changes in anatomy and woody phloem as well as changes in the composition and flow of resins (Sancho-Knapik et al., 2017; Gershenzon and Dudareva, 2007; Phillips and Croteau, 1999). Resins are complex mixtures of different classes of terpenoids containing the concentrations of monoterpenoids and diterpene acids, and small quantities of sesquiterpenoids (Langenheim, 1994). As the largest class of natural compounds, terpenes are implicated in multiple ecological roles; however, many of these compounds serve primarily as defence substances since they show antimicrobial, fungistatic and insecticidal properties (Cobb et al., 1968; Langenheim, 1994).

Heterobasidion infection in Norway spruce is challenged through accumulation of terpenoid classes and phenolic compounds at the site of infection (Arnerup et al., 2011). The formation of this reaction zone can be expensive in terms of carbon (Oliva et al., 2012), thus inducing growth reductions on attacked trees (Oliva et al., 2010). Moreover carbon-expensive barriers may also imply reduced physiological capacity of trees to cope with drought stress. In addition, genotypic differences in Norway spruce can be expressed in growth traits (Ununger et al., 1988) and drought sensitivity (Karlsson et al., 1997; Modrzynski and Erikkson, 2002), as well as in infection frequency and mortality rate following inoculation with H. annosum (Swedjemark et al., 2001). Resistance to Heterobasidion in Norway spruce can be detected by inoculation tests (Dimitri, 1994; Swedjemark and Stenlid, 1994), measuring the extent of lesions developing in the wood and inner bark (Swedjemark et al., 1997) or with molecular methods (Hietala et al., 2003; Bodles et al., 2006).

The production of Norway spruce clones showing less susceptibility to the pathogen can be promising in order to reduce losses by this pathogenic fungus (Dimitri, 1994). Among the three species of Heterobasidion, Heterobasidion parviporum Niemela \& Korhonen is the most harmful species attacking with preference Norway spruce (Korhonen, 1978; Niemela and Korhonen, 1998). It causes root and butt rot of established trees. The fungus spread primarily via spores, which settle, germinate and form a mycelium on freshly cut stumps or other recently exposed wood surface. The mycelium then extends into the wood and colonizes the stump or the whole tree, including its roots. It may, then, transfer from infected stumps or trees to healthy trees via contact between roots (Korhonen and Stenlid, 1998).

In order to test the hypothesis that Norway spruce responses to fungal infection differ between clones, and are also affected by environmental conditions, we conducted a controlled environment experiment on seedlings subjected to water deficit (simulating drought stress) and inoculated with an isolate of rot fungus Heterobasidion parviporum in conjunction with wounding to stimulate tree defences.

Understanding resistance or susceptibility to pathogens in host genotypes and metabolic changes associated with fungal infection will provide useful information when breeding for resistance against Heterobasidion spp. in Norway spruce. The specific objective of this study was to characterize the effects of drought stress treatment and $H$. parviporum infection on defensive monoterpenes in Norway spruce clones, exploring the mechanisms of chemical resistance to the attack of this pathogenic fungus in plants under drought stress conditions.

\section{Material and methods}

\subsection{Plant material}

Two clones c171 and c386 of Norway spruce produced at the Forestry Research Institute of Sweden (SkogForsk) were selected for the experiment. According to preliminary tests (B. Karlsson, personal communication), clone c386 was considered "relatively resistant", whereas clone c171 was considered "relatively susceptible" to $H$. parviporum infections. Ten 4 -year old ramets of each clone were grown in 4-1 pots, filled with $1 / 3$ peat and $2 / 3$ sand, in a greenhouse at the University of Firenze (Italy, 43 $46^{\prime} 55.44^{\prime \prime} \mathrm{N}, 11^{\circ} 13^{\prime} 5.41^{\prime \prime} \mathrm{E}$ ); the average greenhouse temperature was $20^{\circ} \mathrm{C}$ during the day and $10^{\circ} \mathrm{C}$ during the night, ramets were exposed to natural light, $11 \mathrm{hday}^{-1}$. Prior to inoculation all ramets were in good condition and experienced identical light and temperature treatment.

\subsection{Experimental treatment}

Inoculation by an Italian isolate of $H$. parviporum was performed on two-year-old branches of each seedling (regularly watered to field capacity or drought-stressed). The inoculations were conducted on day 0 (21 October) (0 days after treatment: DAT) on two branches of each seedling by applying a fresh Heterobasidion colony plug, grown on malt/ agar $1.5 \%$, on a small wound ( $5 \mathrm{~mm}$ long) made in the bark, following to Swedjemark et al. (2001). Other two branches for each seedling, were wounded and inoculated with sterile water-agar.

Seedlings were randomly assigned to one of the two treatment groups: (1) five seedlings for each clone were well-watered (well-watered seedlings: W seedlings) to field capacity each day; (2) starting from a well-watered condition, on day 0 water was withheld from five drought-stressed seedlings (drought-stressed seedlings: D seedlings) for each clone for a 22-day period (until stomata closed). After 22 days from beginning the treatment (day after treatment: DAT), D seedlings were re-irrigated to avoid irreversible damage. Samples from D and W plants were collected 22 DAT, at the peak stress, and 44 DAT, at the end of the experiment.

Seedlings were arranged in a complete randomized block design in the greenhouse and pots were rotated every 3 days throughout the experiment to remove potential microclimate effects. 


\subsection{Fungal growth}

Samples for fungal growth measurements were collected 22 and 44 DAT from W and D seedlings. Fungal wood colonization was measured by dividing the branches into $5 \mathrm{~mm}$ pieces from the inoculum point. The branch pieces were put in Petri plates (water/agar substrate) in a constant temperature chamber at $20 \pm 2{ }^{\circ} \mathrm{C}$. After 4 days, each piece was observed at the dissecting microscope, looking for conidial form.

\subsection{Physiological response}

Photosynthetic parameters and water relations were measured to monitor drought stress development $(1,4,8,11,15,18$ and 23 DAT) and during recovery from drought stress $(25,30$ and 44 DAT), on non-inoculated 2-year old branches of five replicate plants for each spruce clone - water treatment combination.

Predawn water potential was determined using a custom-made pressure chamber.

Gas exchange was measured between 9:00 and 12:00 using a portable infra-red gas analysis system (Ciras-1, PP-System, UK) equipped with conifer cuvette with light unit $\left(1000 \mu \mathrm{mol}\right.$ photons $\left.\mathrm{m}^{-2} \mathrm{~s}^{-1}\right)$; ambient $\mathrm{CO}_{2}$ was set at $370 \mathrm{ppm}$ and air humidity at $80 \%$. At the end of the experiment, the shoots were harvested and the projected needle areas measured with a Licor-3000 planimeter. Gas exchange measurements were expressed on a projected needle area basis.

Chlorophyll fluorescence was determined on intact needles between 9:00 and 12:00 with a portable PAM 2000 fluorimeter (Walts-Effeltrich Co., Germany), on the same plant material used for gas exchange measurements. Needles were sampled at the end of the dark period $(8 \mathrm{~h})$. Minimal (dark) fluorescence $\left(\mathrm{F}_{0}\right)$ yield was obtained upon excitation with a weak measuring beam from a pulse light-emitting diode, while maximal fluorescence yield $\left(\mathrm{F}_{\mathrm{m}}\right)$ was determined after exposure to a $0.8 \mathrm{~s}$ saturating pulse of white light. Therefore, the potential photochemical efficiency of PSII $\left(\mathrm{F}_{\mathrm{v}} / \mathrm{F}_{\mathrm{m}}\right)$ was estimated as the ratio of variable $\left(F_{v}\right)$ to maximal fluorescence yield (Schreiber et al., 1994).

\subsection{Monoterpenes}

Cortical samples were taken from the zones inoculated with $H$. parviporum (I) and sterile water (L) $(0.5 \mathrm{~cm}$ above and below inoculation point), as well as bark from non-inoculated branches (C) and used for terpene analysis.

Cortical samples were sampled from branches of five ramets of each clone on 0 DAT prior to inoculation, to detect the constitutive monoterpene profiles. Sampling from $H$. parviporum inoculum zone was performed $22 \mathrm{DAT}$ at the peak stress (drought + fungal), from five $\mathrm{W}$ and five D seedlings of each clone and repeated at the end of the experimental period (44 DAT). Sampling from sterile water inoculation zone and cortical tissues from non-inoculated branches in D and W seedlings was carried out on the same dates. Approximately $0.04-0.02 \mathrm{~g}$ fresh weight per sample was ground and placed in glass vials with, respectively, $1-0.5 \mathrm{ml}$ pentane with tridecane as an internal standard ( $0.2 \mathrm{~g}$ tridecane in $250 \mathrm{ml}$ pentane).

Each vial was sealed with a Teflon septum and crimped with an aluminium cap. The vials were shaken for $24 \mathrm{~h}$ and filtered using FP 30/ 0.45 CA-S filters with $0.2 \mu \mathrm{m}$ pores (Schleicher and Schuell), and then stored at $-20^{\circ} \mathrm{C}$ until analysis.

Monoterpenes were analyzed by gas chromatography-flame ionization detection (GC-FID) with a Perkin-Elmer Autosystem XL GC, and enantiomeric monoterpenes were separated on a $30 \mathrm{~m}$ Cyclodex-B capillary column, 0.25-mm-diameter, ( $\mathrm{J}$ \& W Scientific, CA). Analysis was carried out under the following conditions: $\mathrm{H}_{2}$ (carrier gas) at $69 \mathrm{kPa}$; injector temperature at $230^{\circ} \mathrm{C}$; detector temperature at $250{ }^{\circ} \mathrm{C}$. The oven temperature programming started at $4{ }^{\circ} \mathrm{C}$ (isothermal, $5 \mathrm{~min}$ ), and increased to $200{ }^{\circ} \mathrm{C}$, at $1.5^{\circ} \mathrm{C} \mathrm{min}^{-1}$, then to $220^{\circ} \mathrm{C}$, at $20^{\circ} \mathrm{C} \mathrm{min}^{-1}$; the final temperature of $220^{\circ} \mathrm{C}$ was maintained for $5 \mathrm{~min}$. Monoterpenes were identified by comparison of retention times with those of standards under the same conditions. Absolute amounts of terpenoids (total concentrations) were determined by comparison with the tridecane internal standard and expressed as $\mathrm{mg} \mathrm{g}^{-1}$ dry weight (DW). The relative amount (proportions or percentages) of each monoterpene comprising the oleoresin mixtures was expressed as a percentage of total monoterpenes (monoterpene profiles).

\subsection{Statistical analysis}

Differences among fungal colonization in the wood were determined using an ANOVA and Tuckey post-hoc test.

Ecophysiological traits measured on sampling times were not normally distributed (Kolmogorov-Smirnov one-sample test) and were therefore analysed using the Friedman test, the nonparametric analogue of repeated measures analyses of variance. Analysis of covariance (ANCOVA) was performed to test for equality of correlation coefficients.

Relative contents (percentages or proportions) of monoterpenes (monoterpene profiles) and total monoterpene concentrations in each sample were calculated and compared among disease-injury categories and water-regime conditions of the plants. Normality was not achieved after an arcsine square-root transformation, so tests were performed using the non-parametric Kruskal-Wallis rank-sum test followed by the Mann-Whitney $U$ Test for multiple comparisons. Monoterpenes measured on sampling times were not normally distributed (KolmogorovSmirnov one-sample test) and were analysed using the Friedman test.

Differences were accepted when significant at the 5\% level. Statistical analyses were performed using SYSTAT 12.0 software (Systat Software Inc., USA).

\section{Results}

\subsection{Fungal growth}

No dead branches or visible lesions were recorded after inoculation. The length of fungal colonization 44 DAT differed between D and W ramets of both clones. The fungal growth in "relatively susceptible" c171, after six weeks of incubation, ranged between 10 and $40 \mathrm{~mm}$ in D plants and 27-72 mm in W ramets; whilst, in "relatively resistant" c386 values were 19-27 and 36-70 mm, respectively. Differences between the clones were not significant. Fungal growth into the stem was generally higher in W (from $27 \mathrm{~mm}$ to $72 \mathrm{~mm}$ ) than in D (from $10 \mathrm{~mm}$ to $40 \mathrm{~mm}$ ) plants. Statistically different fungal growth was observed between the treatments 44 DAT, while no difference was observed 22 DAT.

\subsection{Physiological response}

Predawn water potential $\left(\Psi_{\mathrm{pd}}\right)$ stomatal conductance $\left(G_{\mathrm{s}}\right)$, net photosynthetic rate $\left(P_{n}\right)$, and potential photochemical efficiency of PSII $\left(\mathrm{F}_{\mathrm{v}} / \mathrm{F}_{\mathrm{m}}\right)$ were significantly affected during the course of the drought stress-recovery cycle (Table 1 ).

Predawn water potential did not differ significantly between clones, in $\mathrm{W}$ plants, averaging approximately $-0.7 \mathrm{MPa}$ throughout the experiment (Fig. 1A). Predawn water potential decreased progressively in D plants from day 7. Differences in $\Psi_{\mathrm{pd}}$ between the two clones (D plants) were significant only $14\left(n=7, \chi^{2}=4.6, p<0.05\right)$ and 23 DAT $\left(n=6, \chi^{2}=3.9, p<0.05\right)$. The decrease in $\Psi_{\mathrm{pd}}$ was stronger in "relatively susceptible" c171 than in "relatively resistant" c386 23 DAT, reaching a minimum value of $-3.3 \mathrm{MPa}$ and $-1.4 \mathrm{MPa}$ (on average), respectively. However, soon after re-watering (2 days, particularly in "relatively resistant" c386), $\Psi_{\mathrm{pd}}$ recovered to values similar to those observed before the application of the drought stress treatment.

In W plants values of $G_{\mathrm{s}}$ and $P_{\mathrm{n}}$ were more often higher in "relatively resistant" c386 (averaging $102 \mathrm{mmol} \mathrm{m}^{-2} \mathrm{~s}^{-1}$ and $9.6 \mu \mathrm{mol} \mathrm{m}^{-2} \mathrm{~s}^{-1}$, 
Table 1

Statistical results of the Friedman test examining variation in predawn water potential $\left(\Psi_{\mathrm{pd}}\right)$, stomatal conductance $\left(G_{\mathrm{s}}\right)$, net photosynthetic rate $\left(P_{\mathrm{n}}\right)$, relative fluorescence $\left(\mathrm{F}_{\mathrm{v}} / \mathrm{F}_{\mathrm{m}}\right)$ in seedlings of two Norway spruce clones during the course of a drought stress-recovery cycle (degrees of freedom $=8 ; 9$ for $\psi_{\mathrm{pd}}$ ): $\chi^{2}$ value; $p$-value where ${ }^{*} p<0.05$. NS: not significant. Well-watered (W) plants; drought-stressed (D) plants.

\begin{tabular}{lllll}
\hline Variable & Clone & N. Samples & Water regime & $\chi^{2}(p)$ \\
\hline$\psi_{\mathrm{pd}}[\mathrm{MPa}]$ & $\mathrm{c} 171$ & 27 & $\mathrm{~W}$ & $12.09 \mathrm{NS}$ \\
& $\mathrm{c} 171$ & 29 & $\mathrm{D}$ & $16.88^{*}$ \\
& $\mathrm{c} 386$ & 27 & $\mathrm{~W}$ & $12.69 \mathrm{NS}$ \\
& $\mathrm{c} 386$ & 28 & $\mathrm{D}$ & $18.15^{*}$ \\
$G_{\mathrm{s}}\left[\mathrm{mmol}\left(\mathrm{CO}_{2}\right) \mathrm{m}^{-2} \mathrm{~s}^{-1}\right]$ & $\mathrm{c} 171$ & 41 & $\mathrm{~W}$ & $11.67 \mathrm{NS}$ \\
& $\mathrm{c} 171$ & 36 & $\mathrm{D}$ & $16.00^{*}$ \\
& $\mathrm{c} 386$ & 41 & $\mathrm{~W}$ & $10.78 \mathrm{NS}$ \\
& $\mathrm{c} 386$ & 38 & $\mathrm{D}$ & $17.67 *$ \\
$P_{\mathrm{n}}\left[\mu \mathrm{mol}\left(\mathrm{CO}_{2}\right) \mathrm{m}^{-2} \mathrm{~s}^{-1}\right]$ & $\mathrm{c} 171$ & 42 & $\mathrm{~W}$ & $10.67 \mathrm{NS}$ \\
& $\mathrm{c} 171$ & 34 & $\mathrm{D}$ & $15.22^{*}$ \\
& $\mathrm{c} 386$ & 41 & $\mathrm{~W}$ & $13.56 \mathrm{NS}$ \\
& $\mathrm{c} 386$ & 36 & $\mathrm{D}$ & $14.22^{*}$ \\
$\mathrm{~F}_{\mathrm{v}} / \mathrm{F}_{\mathrm{m}}$ & $\mathrm{c} 171$ & 41 & $\mathrm{~W}$ & $6.33 \mathrm{NS}$ \\
& $\mathrm{c} 171$ & 37 & $\mathrm{D}$ & $17.89^{*}$ \\
& $\mathrm{c386}$ & 42 & $\mathrm{~W}$ & $10.93 \mathrm{NS}$ \\
& $\mathrm{c386}$ & 40 & $\mathrm{D}$ & $17.25^{*}$ \\
\hline
\end{tabular}

respectively) than in "relatively susceptible" c171 (averaging $83 \mathrm{mmol} \mathrm{m}^{-2} \mathrm{~s}^{-1}$ and $8.0 \mu \mathrm{mol} \mathrm{m} \mathrm{m}^{-2} \mathrm{~s}^{-1}$, respectively).Gas exchange started decreasing after the imposition of drought stress, following $\Psi_{\mathrm{pd}}$ (Fig. 1B, C). After imposing drought stress, $G_{\mathrm{s}}$ and $P_{\mathrm{n}}$ decreased more rapidly in "relatively resistant" c386 than in "relatively susceptible" c171, reaching stable low values after 8-11 days (minimum of 14.0 and $6.9 \mathrm{mmol} \mathrm{m}^{-2} \mathrm{~s}^{-1}$, and 1.02 and $0.96 \mu \mathrm{mol} \mathrm{m}^{-2} \mathrm{~s}^{-1}$, respectively). The recovery of $G_{\mathrm{s}}$ and $P_{\mathrm{n}}$ after re-watering of D plants was slow in both
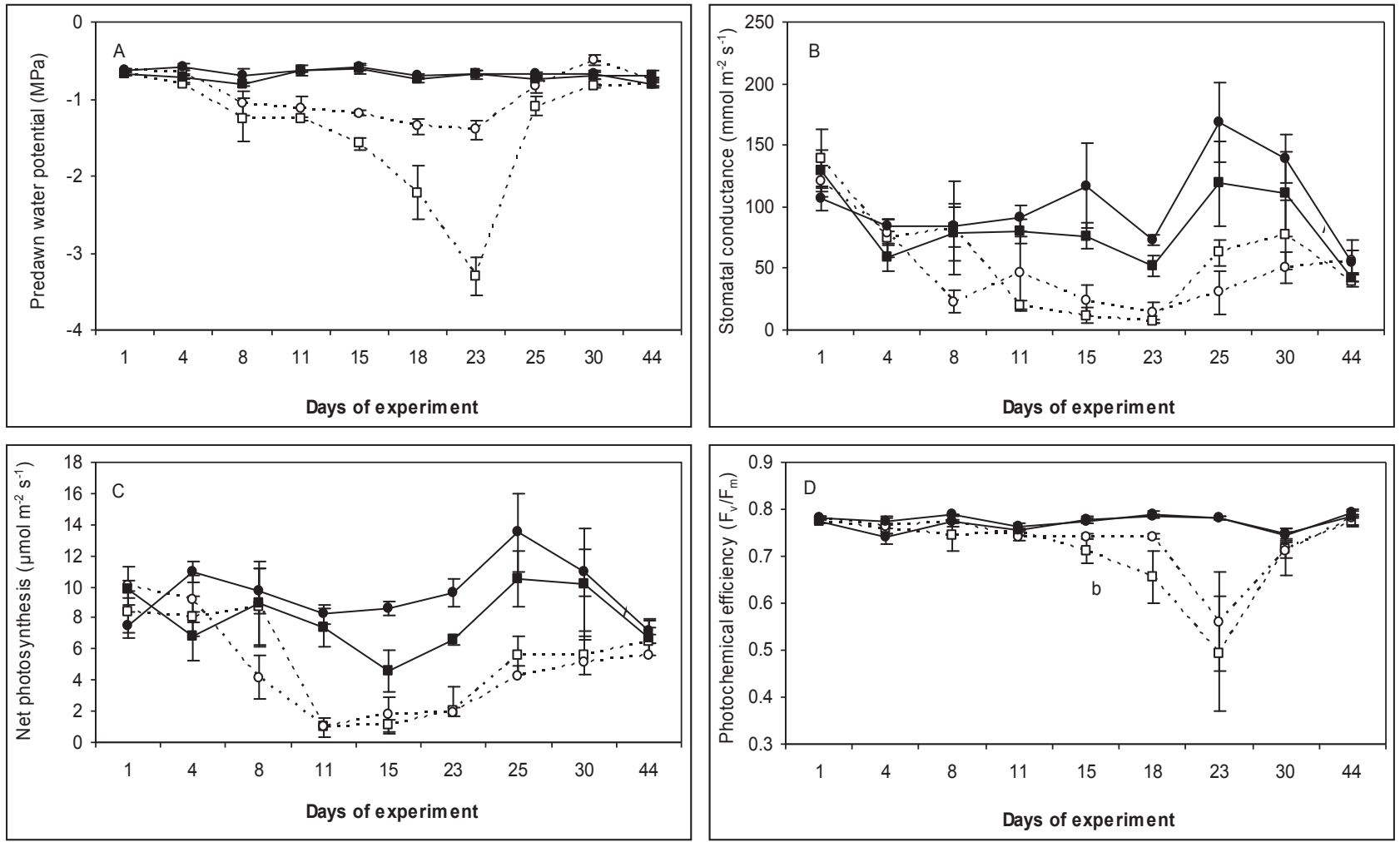

Fig. 1. Physiological responses of two clones of $P$. abies to water stress-recovery cycle. On day 0 all seedlings were watered to field capacity. Seedlings were then either watered daily (W: well-watered) or not watered (D: drought-stressed). Stressed plants were irrigated daily since day 23. Inoculations with $H$. parviporum were made on day 0 . Values are means \pm SE $(n=3-5)$. Filled circle and solid line is c386 (W), hollow circle and dashed line is c386 (D), Filled square and solid line is c171 (W), hollow squares and dotted line is c171 (D). 

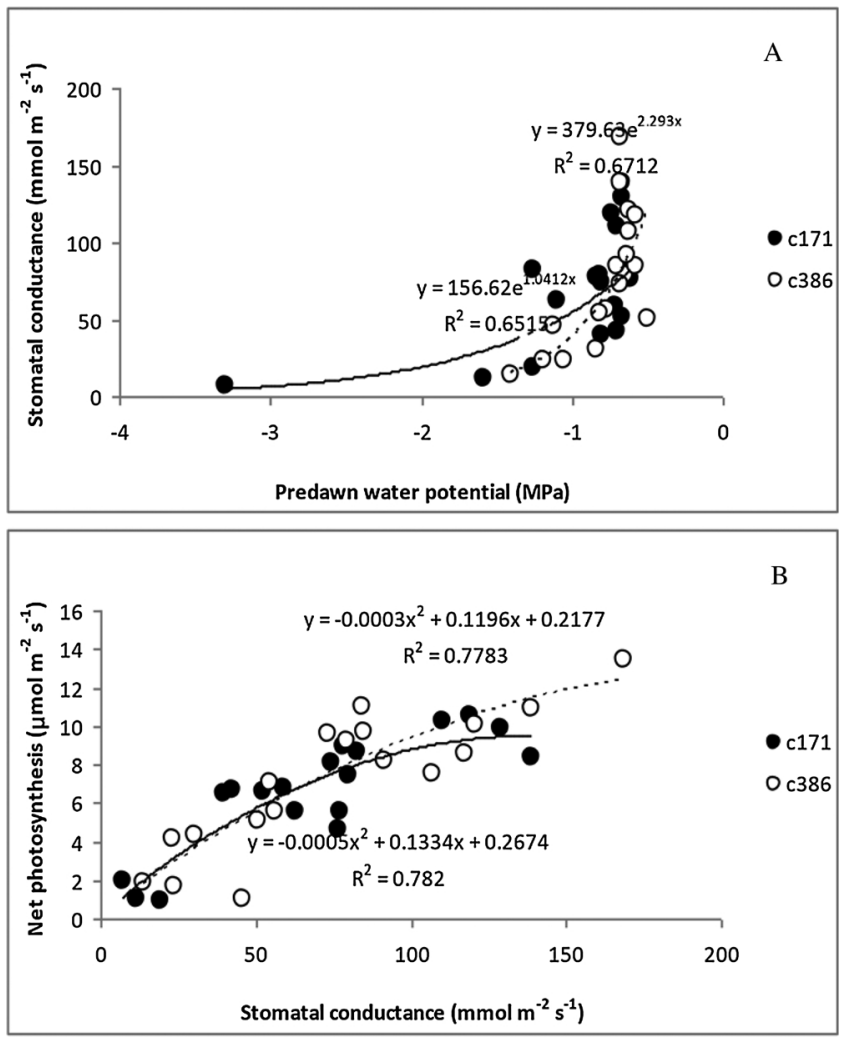

Fig. 2. Regressions between stomatal conductance and (A) predawn shoot water potential, and (B) net photosynthesis of seedlings of Norway spruce exposed to a period of drought stress. The curves were fitted using exponential or non-linear, second order regressions $(p<0.001)$.

c171 ramets and five c386 ramets: c386 characterized by high relative contents of $\delta$-3-carene $\left(\mathrm{p}<0.01, \quad \chi^{2}=5.95\right), \quad(-)$-limonene $\left(\mathrm{p}<0.01, \chi^{2}=6.05\right), \gamma$-terpinene $\left(\mathrm{p}<0.02, \chi^{2}=5.21\right)$, and terpinolene ( $\left.\mathrm{p}<0.01, \chi^{2}=6.00\right)$; while, high proportions of $(-)$ - $\alpha$-pinene $\left(\mathrm{p}<0.01, \chi^{2}=6.16\right),(+)-\alpha$-pinene $\left(\mathrm{p}<0.01, \chi^{2}=6.00\right),(-)-\beta-$ pinene, $\quad\left(\mathrm{p}<0.01, \quad \chi^{2}=6.35\right)$ and $(+)$-limonene $(\mathrm{p}<0.01$, $\chi^{2}=6.53$ ), were observed in c171 (Fig. 3).

Mean relative amounts \pm standard error of monoterpenes within spruce clone, disease treatment, sampling time and water status condition are reported in Table 2.

C samples on the same day did not show significant variations between D and W plants, in the relative content of all the monoterpenes, except $p$-cymene in both clones and terpinolene in c171 seedlings 22 DAT (Table 3).

Also, sampling time (0 DAT, 22 DAT and 44 DAT) did not significantly change the relative contents of all the monoterpenes in $\mathrm{C}$ samples of W seedlings within the same spruce clone, except for sabinene in "relatively susceptible" c171 as indicated by Friedman test (Supplementary material Table S1).

Kruskal-Wallis ANOVA between disease treatments within the same spruce clone, water regime and sampling time (Supplementary material Table S2) showed that alterations in the relative contents of several monoterpenes were significantly affected by wounding and inoculation with $H$. parviporum.

With specific regard to 171 , proportions of $\delta$-3-carene, (-)-limonene and terpinolene increased in all I tissues; the relative contents of myrcene was lower in $\mathrm{L}$ samples than in the other treatments in $\mathrm{W}$ and D plants sampled respectively 22 DAT and 44 DAT; proportions of sabinene and $\gamma$-terpinene were higher in I tissues than in the other treatments of $\mathrm{W}$ plants sampled 44 DAT.

The relative contents of (-)- $\alpha$-pinene and (+)- $\alpha$-pinene decreased in I tissues in both $\mathrm{W}$ and D plants sampled 22 DAT and W plants sampled 44 DAT; inoculation with $H$. parviporum also induced a decrease in proportions of $(-)-\beta$-pinene in $\mathrm{W}$ plants on both sampling times.

Proportions of myrcene decreased in L samples in W and D plants sampled, respectively, 22 and 44 DAT.

With specific regard to c386, the proportions of (-)-limonene increased in all I tissues; higher relative contents of $(+)$ - $\alpha$-pinene were detected in I and L tissues than in C samples in D plants 44 DAT; the relative content of myrcene was higher in I samples than in the other treatments in W plants sampled 22 DAT and 44 DAT, while it decreased

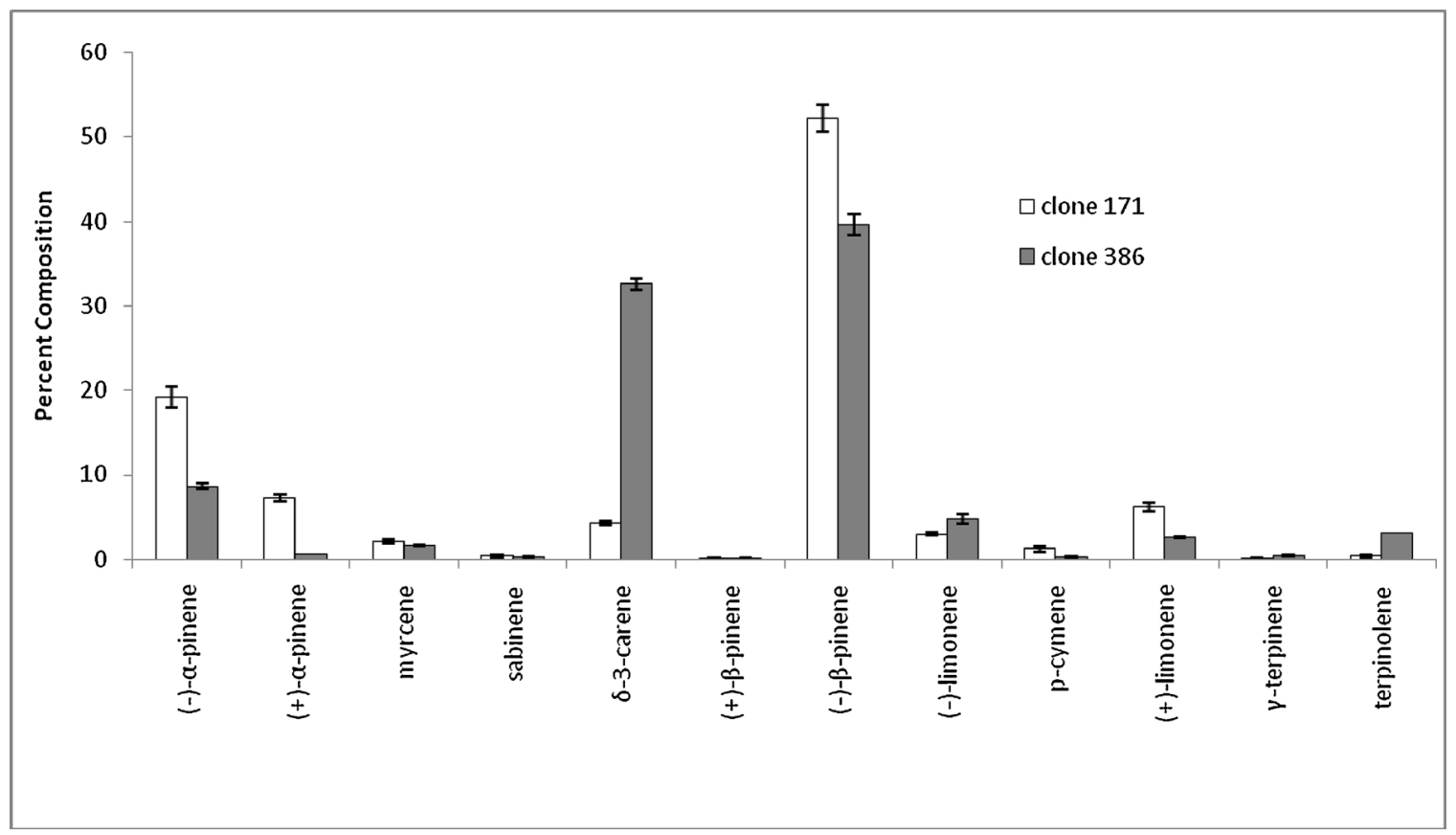

Fig. 3. Monoterpene profiles (bars indicate means \pm standard error) obtained from two Norway spruce clones on day 0: "relatively susceptible" clone 171 and "relatively resistant" clone 386 to attack by Heterobasidion parviporum. 
Table 2

Relative contents of monoterpenes (means \pm standard error) of two Norway spruce clones in response to Heterobasidion annosum. Treatments: drought-stressed plants (D); well-watered (W); cortical tissues from non-inoculated branches (C); wounded plus inoculated with H. parviporum (I); wounded only (L). Different letters indicate significantly different disease treatment means (Mann-Whitney test) within the same Norway spruce clone, water regime, and sampling time. Other monoterpenes ( $\alpha$-thujene, camphene, $\alpha$-phellandrene, tricyclene, $\beta$-phellandrene, cineole, terpineol, $\alpha$-terpinene, bornylacetate) and an unknown compound representing about $4-8 \%$ of total monoterpenes were omitted in the table .

\begin{tabular}{|c|c|c|c|c|c|c|}
\hline \multirow[t]{4}{*}{ Monoterpene } & \multicolumn{3}{|l|}{$\mathrm{D}$} & \multicolumn{3}{|l|}{$\mathrm{W}$} \\
\hline & I & $\mathrm{L}$ & $\mathrm{C}$ & I & $\mathrm{L}$ & $\mathrm{C}$ \\
\hline & \multicolumn{6}{|l|}{ Clone 171} \\
\hline & \multicolumn{6}{|l|}{ Day 22} \\
\hline (-)- $\alpha$-pinene & $16.30 \pm 0.63 \mathrm{a}$ & $18.56 \pm 0.72 b$ & $19.71 \pm 0.24 \mathrm{~b}$ & $12.75 \pm 0.32 \mathrm{a}$ & $19.92 \pm 0.91 \mathrm{~b}$ & $19.14 \pm 1.22 \mathrm{~b}$ \\
\hline$(+)$ - $\alpha$-pinene & $4.27 \pm 0.85 \mathrm{a}$ & $8.34 \pm 1.22 \mathrm{~b}$ & $8.59 \pm 1.49 \mathrm{~b}$ & $1.53 \pm 0.30 \mathrm{a}$ & $6.35 \pm 0.98 \mathrm{~b}$ & $7.22 \pm 0.45 b$ \\
\hline myrcene & $2.31 \pm 0.10$ & $2.36 \pm 0.56$ & $2.27 \pm 0.14$ & $2.75 \pm 0.07 \mathrm{~b}$ & $1.49 \pm 0.54 \mathrm{a}$ & $2.09 \pm 0.22 \mathrm{~b}$ \\
\hline sabinene & $0.11 \pm 0.07$ & $0.31 \pm 0.16$ & Trace & $0.13 \pm 0.03$ & $0.10 \pm 0.10$ & $0.37 \pm 0.09$ \\
\hline$\delta$-3-carene & $10.58 \pm 1.81 \mathrm{~b}$ & $5.31 \pm 1.89 \mathrm{a}$ & $4.09 \pm 0.66 \mathrm{a}$ & $18.82 \pm 0.41 \mathrm{~b}$ & $5.26 \pm 0.99 \mathrm{a}$ & $4.33 \pm 0.23 \mathrm{a}$ \\
\hline$(+)$ - $\beta$-pinene & $0.63 \pm 0.16$ & $0.98 \pm 0.09$ & $0.20 \pm 0.20$ & Trace & $0.15 \pm 0.15$ & Trace \\
\hline$(-)-\beta$-pinene & $45.77 \pm 1.61$ & $48.15 \pm 0.38$ & $51.91 \pm 1.43$ & $36.95 \pm 0.35 \mathrm{a}$ & $52.24 \pm 2.7 \mathrm{~b}$ & $52.20 \pm 1.6 \mathrm{~b}$ \\
\hline (-)-limonene & $7.45 \pm 1.55 b$ & $3.55 \pm 0.86 \mathrm{ab}$ & $2.80 \pm 0.46 \mathrm{a}$ & $15.32 \pm 1.11 \mathrm{~b}$ & $2.23 \pm 0.76 \mathrm{a}$ & $2.98 \pm 0.14 \mathrm{a}$ \\
\hline p-cymene & $0.74 \pm 0.12$ & $0.90 \pm 0.54$ & $0.19 \pm 0.19$ & $0.60 \pm 0.05 \mathrm{a}$ & $0.20 \pm 0.20 \mathrm{a}$ & $1.22 \pm 0.28 b$ \\
\hline$(+)$-limonene & $6.11 \pm 0.41$ & $5.80 \pm 0.31$ & $6.39 \pm 0.24$ & $5.56 \pm 0.23$ & $6.70 \pm 0.94$ & $6.17 \pm 0.52$ \\
\hline$\gamma$-terpinene & $0.04 \pm 0.04$ & Trace & Trace & $0.28 \pm 0.07 \mathrm{~b}$ & Trace & Trace \\
\hline \multirow[t]{2}{*}{ terpinolene } & $0.94 \pm 0.17 \mathrm{~b}$ & $0.35 \pm 0.18 \mathrm{~b}$ & Trace a & $1.81 \pm 0.06 \mathrm{~b}$ & $0.16 \pm 0.16 \mathrm{a}$ & $0.37 \pm 0.10 \mathrm{a}$ \\
\hline & \multicolumn{6}{|l|}{ Day 44} \\
\hline$(-)$ - $\alpha$-pinene & $15.53 \pm 0.81 \mathrm{a}$ & $19.29 \pm 0.85 b$ & $19.17 \pm 0.69 \mathrm{~b}$ & $11.87 \pm 0.70 \mathrm{a}$ & $17.55 \pm 0.82 b$ & $19.77 \pm 1.75 \mathrm{~b}$ \\
\hline$(+)$ - $\alpha$-pinene & $3.84 \pm 0.88 \mathrm{a}$ & $7.22 \pm 0.55 b$ & $7.83 \pm 0.71 \mathrm{~b}$ & $1.05 \pm 0.12 \mathrm{a}$ & $7.43 \pm 0.88 b$ & $7.57 \pm 2.2 \mathrm{~b}$ \\
\hline myrcene & $2.59 \pm 0.16 \mathrm{~b}$ & $1.72 \pm 0.29 \mathrm{a}$ & $2.17 \pm 0.04 \mathrm{~b}$ & $2.88 \pm 0.28$ & $2.19 \pm 0.22$ & $2.44 \pm 0.44$ \\
\hline sabinene & $0.17 \pm 0.05$ & $0.19 \pm 0.11$ & Trace & $0.20 \pm 0.02 \mathrm{~b}$ & $0.18 \pm 0.18 \mathrm{~b}$ & Trace a \\
\hline$\delta$-3-carene & $12.57 \pm 2.16 b$ & $4.66 \pm 2.5 \mathrm{a}$ & $4.22 \pm 1.14 \mathrm{a}$ & $22.83 \pm 1.79 \mathrm{c}$ & $5.1 \pm 0.69 \mathrm{~b}$ & $4.46 \pm 0.46 \mathrm{a}$ \\
\hline$(+)-\beta$-pinene & $0.63 \pm 0.17$ & $1.00 \pm 0.05$ & $0.32 \pm 0.32$ & Trace & $0.4 \pm 0.24$ & Trace \\
\hline$(-)-\beta$-pinene & $43.90 \pm 1.50$ & $50.77 \pm 2.03$ & $51.26 \pm 1.70$ & $27.07 \pm 5.16 \mathrm{a}$ & $53.50 \pm 1.90 \mathrm{~b}$ & $53.91 \pm 3.97 b$ \\
\hline (-)-limonene & $9.03 \pm 1.67 \mathrm{~b}$ & $2.56 \pm 0.21 \mathrm{a}$ & $2.87 \pm 0.55 \mathrm{a}$ & $21.54 \pm 1.89 \mathrm{c}$ & $4.36 \pm 0.32 \mathrm{~b}$ & $1.10 \pm 0.69 \mathrm{a}$ \\
\hline p-cymene & $0.58 \pm 0.02$ & $1.47 \pm 0.75$ & $0.76 \pm 0.41$ & $0.63 \pm 0.04$ & $0.23 \pm 0.14$ & $0.19 \pm 0.19$ \\
\hline$(+)$-limonene & $6.51 \pm 0.51$ & $4.38 \pm 1.46$ & $6.10 \pm 0.22$ & $5.42 \pm 0.63$ & $6.38 \pm 0.60$ & $6.20 \pm 1.36$ \\
\hline$\gamma$-terpinene & $0.10 \pm 0.04$ & Trace & Trace & $0.27 \pm 0.03 \mathrm{~b}$ & Trace a & Trace a \\
\hline \multirow[t]{3}{*}{ terpinolene } & $1.26 \pm 0.23 \mathrm{~b}$ & $0.24 \pm 0.24 \mathrm{a}$ & $0.18 \pm 0.18 \mathrm{a}$ & $2.04 \pm 0.23 b$ & $0.14 \pm 0.14 \mathrm{a}$ & Trace a \\
\hline & \multicolumn{6}{|l|}{ Clone 386} \\
\hline & \multicolumn{6}{|l|}{ Day 22} \\
\hline$(-)$ - $\alpha$-pinene & $8.83 \pm 1.53 \mathrm{a}$ & $14.6 \pm 1.44 \mathrm{~b}$ & $8.02 \pm 0.34 \mathrm{a}$ & $7.11 \pm 0.16 \mathrm{a}$ & $11.44 \pm 0.86 \mathrm{~b}$ & $8.62 \pm 0.3 \mathrm{a}$ \\
\hline$(+)$ - $\alpha$-pinene & $0.33 \pm 0.09$ & $0.33 \pm 0.20$ & $0.49 \pm 0.04$ & $0.43 \pm 0.09$ & $0.63 \pm 0.33$ & $0.55 \pm 0.06$ \\
\hline myrcene & $1.63 \pm 0.43 \mathrm{~b}$ & $0.79 \pm 0.35 \mathrm{a}$ & $1.74 \pm 0.06 \mathrm{~b}$ & $2.57 \pm 0.11 \mathrm{~b}$ & $1.49 \pm 0.75 \mathrm{a}$ & $1.65 \pm 0.07 \mathrm{a}$ \\
\hline sabinene & $0.15 \pm 0.05 \mathrm{a}$ & $0.20 \pm 0.14 \mathrm{~b}$ & $0.48 \pm 0.04 \mathrm{~b}$ & $0.19 \pm 0.06$ & $0.16 \pm 0.16$ & $0.31 \pm 0.08$ \\
\hline$\delta$-3-carene & $34.18 \pm 0.82$ & $35.13 \pm 3.71$ & $32.26 \pm 0.84$ & $36.00 \pm 1.47$ & $28.28 \pm 2.36$ & $32.56 \pm 0.72$ \\
\hline$(+)$ - $\beta$-pinene & Trace & Trace & Trace & Trace & Trace & Trace \\
\hline$(-)-\beta$-pinene & $28.06 \pm 4.23$ & $33.77 \pm 3.31$ & $38.17 \pm 1.05$ & $21.67 \pm 0.76 \mathrm{a}$ & $39.98 \pm 1.87 \mathrm{c}$ & $29.61 \pm 1.24 \mathrm{~b}$ \\
\hline (-)-limonene & $13.07 \pm 2.98 \mathrm{c}$ & $2.67 \pm 0.71 \mathrm{a}$ & $4.86 \pm 0.46 \mathrm{~b}$ & $16.95 \pm 1.04 \mathrm{~b}$ & $6.49 \pm 1.61 \mathrm{a}$ & $4.78 \pm 0.53 \mathrm{a}$ \\
\hline p-cymene & $0.46 \pm 0.13 \mathrm{~b}$ & $0.07 \pm 0.07 \mathrm{a}$ & $0.57 \pm 0.03 \mathrm{~b}$ & $0.73 \pm 0.09$ & $0.91 \pm 0.50$ & $0.27 \pm 0.11$ \\
\hline$(+)$-limonene & $2.74 \pm 0.70$ & $3.93 \pm 0.5$ & $2.71 \pm 0.09$ & $3.39 \pm 0.19$ & $3.07 \pm 0.29$ & $2.61 \pm 0.14$ \\
\hline$\gamma$-terpinene & $0.38 \pm 0.09 \mathrm{~b}$ & $0.09 \pm 0.09 \mathrm{a}$ & $0.53 \pm 0.04 \mathrm{~b}$ & $0.48 \pm 0.01$ & $0.14 \pm 0.14$ & $0.44 \pm 0.02$ \\
\hline \multirow[t]{2}{*}{ terpinolene } & $3.04 \pm 0.78 b$ & $2.04 \pm 0.55 \mathrm{a}$ & $3.26 \pm 0.14 \mathrm{~b}$ & $3.77 \pm 0.16 b$ & $1.73 \pm 0.94 \mathrm{a}$ & $3.03 \pm 0.05 b$ \\
\hline & \multicolumn{6}{|l|}{ Day 44} \\
\hline$(-)$ - $\alpha$-pinene & $9.65 \pm 0.39$ & $11.48 \pm 1.13$ & $8.83 \pm 0.82$ & $5.72 \pm 0.3 \mathrm{a}$ & $11.62 \pm 0.94 \mathrm{~b}$ & $8.3 \pm 0.83 b$ \\
\hline$(+)$ - $\alpha$-pinene & $0.60 \pm 0.07 b$ & $0.48 \pm 0.16 \mathrm{~b}$ & Trace a & $0.38 \pm 0.05$ & $0.65 \pm 0.17$ & $0.33 \pm 0.2$ \\
\hline myrcene & $1.77 \pm 0.16$ & $1.11 \pm 0.37$ & $1.84 \pm 0.48$ & $2.43 \pm 0.05 \mathrm{~b}$ & $1.17 \pm 0.34 \mathrm{a}$ & $1.45 \pm 0.51 \mathrm{a}$ \\
\hline sabinene & $0.22 \pm 0.07$ & $0.12 \pm 0.12$ & Trace & $0.16 \pm 0.07 \mathrm{~b}$ & Trace a & Trace a \\
\hline$\delta$-3-carene & $31.49 \pm 1.67$ & $28.01 \pm 0.64$ & $30.31 \pm 1.1$ & $45.06 \pm 1.08 \mathrm{~b}$ & $34.21 \pm 1.52 \mathrm{a}$ & $28.11 \pm 4.68 \mathrm{a}$ \\
\hline$(+)-\beta$-pinene & Trace a & $0.57 \pm 0.19 b$ & Trace a & Trace & $0.31 \pm 0.19$ & Trace \\
\hline$(-)-\beta$-pinene & $34.46 \pm 2.21 \mathrm{a}$ & $43.11 \pm 3.53 b$ & $42.87 \pm 2.78 b$ & $15.22 \pm 1.30 \mathrm{a}$ & $38.88 \pm 2.91 \mathrm{c}$ & $26.77 \pm 3.77 b$ \\
\hline (-)-limonene & $9.26 \pm 2.08 \mathrm{~b}$ & $4.67 \pm 0.9 \mathrm{a}$ & $4.46 \pm 0.58 \mathrm{a}$ & $18.99 \pm 1.20 \mathrm{~b}$ & $3.91 \pm 1.07 \mathrm{a}$ & $6.44 \pm 4.24 \mathrm{a}$ \\
\hline p-cymene & $0.30 \pm 0.10$ & $0.12 \pm 0.12$ & Trace & $0.49 \pm 0.03 \mathrm{~b}$ & $0.07 \pm 0.07 \mathrm{a}$ & Trace a \\
\hline$(+)$-limonene & $3.08 \pm 0.16$ & $2.48 \pm 0.09$ & $2.03 \pm 0.73$ & $2.95 \pm 0.08$ & $2.03 \pm 0.61$ & $2.26 \pm 0.76$ \\
\hline$\gamma$-terpinene & $0.31 \pm 0.1$ & $0.21 \pm 0.12$ & Trace & $0.57 \pm 0.02 \mathrm{~b}$ & Trace a & Trace a \\
\hline terpinolene & $2.84 \pm 0.26$ & $2.33 \pm 0.25$ & $2.29 \pm 0.79$ & $4.03 \pm 0.39 \mathrm{~b}$ & $1.83 \pm 0.52 \mathrm{a}$ & $2.40 \pm 0.85 \mathrm{ab}$ \\
\hline
\end{tabular}

in L samples in D plants sampled 22 DAT. The proportions of sabinene, $\delta$-3-carene, $\gamma$-terpinene, and terpinolene increased more in I tissues than in the other treatments in W plants sampled 44 DAT.

The relative contents of (-)- $\alpha$-pinene increased in all L samples 22 DAT, while it decreased in I tissues in W plants collected 44 DAT.

The relative contents of (-)- $\beta$-pinene increased in L samples, while it decreased in I tissues in W plants sampled 22 DAT and 44 DAT.

The Mann-Whitney $U$ Test within the same clone, indicated that disease treatment and sampling time showed significant variations in the relative content of monoterpenes in response to drought stress (Table 3).

With specific regard to 171 , the relative contents of $(-)$ - $\alpha$-pinene, $(+)$ - $\alpha$-pinene, $(+)-\beta$-pinene, and $(-)-\beta$-pinene in all I tissues were higher in $\mathrm{D}$ than in $\mathrm{W}$ plants, while the proportions of $\delta$-3-carene, $(-)$-limonene, $\gamma$-terpinene, and terpinolene decreased in response to drought stress.

L samples showed lower proportions of $(-)$-limonene and higher relative contents of $p$-cymene in D plants than in W plants 44 DAT. 
Table 3

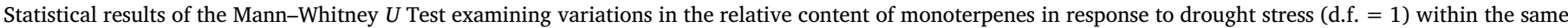

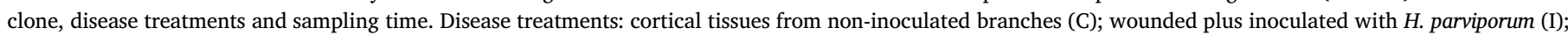
wounded only (L); $p$-value where ${ }^{*} p<0.05$, and ${ }^{* *} p<0.01$. NS: not significant. $\mathrm{N}^{\circ}$ samples: 3-5.

\begin{tabular}{|c|c|c|c|c|c|c|c|}
\hline \multirow[t]{3}{*}{ Monoterpene } & \multirow[t]{3}{*}{ Clone } & \multicolumn{6}{|l|}{$\chi^{2}(p)$} \\
\hline & & \multicolumn{2}{|l|}{$\mathrm{C}$} & \multicolumn{2}{|l|}{$\mathrm{L}$} & \multicolumn{2}{|l|}{ I } \\
\hline & & Day 22 & Day 43 & Day 22 & Day 43 & Day 22 & Day 43 \\
\hline \multirow[t]{2}{*}{$(-)$ - $\alpha$-pinene } & c171 & $3.81 \mathrm{NS}$ & $0.02 \mathrm{NS}$ & $0.50 \mathrm{NS}$ & $2.16 \mathrm{NS}$ & $6.70^{* *}$ & $6.82 * *$ \\
\hline & c386 & $3.15 \mathrm{NS}$ & $0.08 \mathrm{NS}$ & $0.02 \mathrm{NS}$ & $0.54 \mathrm{NS}$ & $0.88 \mathrm{NS}$ & $6.00^{*}$ \\
\hline \multirow[t]{2}{*}{$(+)$ - $\alpha$-pinene } & c171 & 0.27 NS & $0.02 \mathrm{NS}$ & $1.12 \mathrm{NS}$ & $0.15 \mathrm{NS}$ & $6.00^{*}$ & $6.1 * *$ \\
\hline & c386 & $0.27 \mathrm{NS}$ & $2.28 \mathrm{NS}$ & $0.40 \mathrm{NS}$ & $1.83 \mathrm{NS}$ & $0.27 \mathrm{NS}$ & $3.84 *$ \\
\hline \multirow[t]{2}{*}{ myrcene } & c171 & $0.53 \mathrm{NS}$ & $2.04 \mathrm{NS}$ & $0.50 \mathrm{NS}$ & $2.94 \mathrm{NS}$ & $6.34^{* *}$ & $1.32 \mathrm{NS}$ \\
\hline & c386 & 0.27 NS & $0.78 \mathrm{NS}$ & 1.14 NS & $0.14 \mathrm{NS}$ & $4.80^{*}$ & $6.00^{*}$ \\
\hline \multirow[t]{2}{*}{ sabinene } & c171 & $2.81 \mathrm{NS}$ & $0.00 \mathrm{NS}$ & $1.86 \mathrm{NS}$ & $0.34 \mathrm{NS}$ & 0.18 NS & $0.09 \mathrm{NS}$ \\
\hline & c386 & 3.15 NS & $0.00 \mathrm{NS}$ & $0.03 \mathrm{NS}$ & $1.25 \mathrm{NS}$ & $0.41 \mathrm{NS}$ & $4.86^{*}$ \\
\hline \multirow{2}{*}{$\delta$-3-carene } & c171 & $2.45 \mathrm{NS}$ & 3.05 NS & $0.52 \mathrm{NS}$ & $1.50 \mathrm{NS}$ & $5.20^{*}$ & $6.00 * *$ \\
\hline & c386 & 0.27 NS & $0.08 \mathrm{NS}$ & $0.02 \mathrm{NS}$ & $1.40 \mathrm{NS}$ & $0.88 \mathrm{NS}$ & $6.00^{*}$ \\
\hline \multirow[t]{2}{*}{$(+)-\beta$-pinene } & $\mathrm{c} 171$ & $1.00 \mathrm{NS}$ & $1.66 \mathrm{NS}$ & $4.85 \mathrm{NS}$ & $2.23 \mathrm{NS}$ & $5.53^{*}$ & $5.54^{*}$ \\
\hline & c386 & $0.00 \mathrm{NS}$ & $0.00 \mathrm{NS}$ & $0.10 \mathrm{NS}$ & $0.80 \mathrm{NS}$ & $0.10 \mathrm{NS}$ & $0.00 \mathrm{NS}$ \\
\hline \multirow[t]{2}{*}{$(-)-\beta$-pinene } & c171 & $2.81 \mathrm{NS}$ & $0.20 \mathrm{NS}$ & $0.23 \mathrm{NS}$ & $0.96 \mathrm{NS}$ & $5.70^{*}$ & $6.86^{* *}$ \\
\hline & c386 & $0.88 \mathrm{NS}$ & $0.00 \mathrm{NS}$ & $0.56 \mathrm{NS}$ & $0.00 \mathrm{NS}$ & $2.46 \mathrm{NS}$ & $6.00^{*}$ \\
\hline \multirow[t]{2}{*}{$(-)$-limonene } & c171 & $2.81 \mathrm{NS}$ & $1.14 \mathrm{NS}$ & $0.50 \mathrm{NS}$ & $6.00 *$ & $6.20^{* *}$ & $6.00 * *$ \\
\hline & c386 & $0.27 \mathrm{NS}$ & $1.33 \mathrm{NS}$ & $5.00 *$ & $0.06 \mathrm{NS}$ & $1.8 \mathrm{NS}$ & $6.00 *$ \\
\hline \multirow[t]{2}{*}{ p-cymene } & c171 & $4.19 *$ & $1.42 \mathrm{NS}$ & $1.86 \mathrm{NS}$ & $6.21 *$ & $0.53 \mathrm{NS}$ & $0.27 \mathrm{NS}$ \\
\hline & c386 & $3.96^{*}$ & $0.00 \mathrm{NS}$ & $4.36 *$ & $0.11 \mathrm{NS}$ & $3.94^{*}$ & $3.84^{*}$ \\
\hline \multirow[t]{2}{*}{$(+)$-limonene } & c171 & $0.01 \mathrm{NS}$ & $1.82 \mathrm{NS}$ & $0.12 \mathrm{NS}$ & $2.94 \mathrm{NS}$ & $1.32 \mathrm{NS}$ & $2.46 \mathrm{NS}$ \\
\hline & c386 & $0.88 \mathrm{NS}$ & $0.02 \mathrm{NS}$ & $2.36 \mathrm{NS}$ & $0.06 \mathrm{NS}$ & 0.09 NS & $1.50 \mathrm{NS}$ \\
\hline \multirow[t]{2}{*}{$\gamma$-terpinene } & c171 & $0.00 \mathrm{NS}$ & $0.00 \mathrm{NS}$ & $0.15 \mathrm{NS}$ & $0.00 \mathrm{NS}$ & $5.12^{*}$ & $6.90^{* *}$ \\
\hline & c386 & 3.15 NS & $0.00 \mathrm{NS}$ & 0.4 NS & $2.81 \mathrm{NS}$ & $0.10 \mathrm{NS}$ & $4.86^{*}$ \\
\hline \multirow[t]{2}{*}{ terpinolene } & c171 & $5.53^{* *}$ & $1.66 \mathrm{NS}$ & $0.34 \mathrm{NS}$ & $0.11 \mathrm{NS}$ & $6.99 * *$ & $4.80 *$ \\
\hline & c386 & 0.88 NS & 0.02 NS & $0.20 \mathrm{NS}$ & $0.24 \mathrm{NS}$ & 0.27 NS & $4.00^{*}$ \\
\hline
\end{tabular}

With specific regard to clone 386 , proportions of (-)- $\alpha$-pinene, $(+)$ - $\alpha$-pinene, sabinene, and ( - )- $\beta$-pinene, were higher in I tissues in D than in W plants 44 DAT.

The percentages of myrcene and $p$-cymene in all I tissues were lower in $\mathrm{D}$ than $\mathrm{W}$ plants, while the proportions of $\delta$-3-carene, $(-)$-limonene, $\gamma$-terpinene, and terpinolene decreased in response to drought stress only 44 DAT.

The relative content of $(-)$-limonene in $\mathrm{L}$ samples was lower in $\mathrm{D}$ than in $\mathrm{W}$ plants.

Significant variations of the relative contents of monoterpenes were also observed between the two clones within treatments and water plant status (Supplementary material Table S3).

The Friedman Test within the same spruce clone, disease treatment and water status condition showed significant variations in the relative contents of several monoterpenes in response to sampling time (Supplementary material Table S1).

With specific regard to $\mathrm{c} 171$, the proportions of myrcene were higher in I tissues in D plants 44 DAT than 22 DAT.

I tissues in W plants showed higher percentages of $(-)$ - $\beta$-pinene and lower proportions of sabinene, $\delta$-3-carene, $(-)$-limonene 22 DAT than 44 DAT.

L samples in W plants showed lower relative content of (-)-limonene 22 DAT than 44 DAT.

With specific regard to $\mathrm{c} 386$, the proportion of $\delta$-3-carene was higher in I tissues in D plants 22 DAT than 44 DAT.

$\mathrm{L}$ samples in D plants showed lower percentages of $(-)$ - $\beta$-pinene and (-)-limonene 22 DAT than 44 DAT.

I tissues in $\mathrm{W}$ plants showed higher proportions of $(-)$ - $\alpha$-pinene, sabinene, and (-)- $\beta$-pinene, and lower percentages of $\delta$-3-carene, and $\gamma$-terpinene 22 DAT than 44 DAT.

\subsubsection{Total concentrations of monoterpenes (TCM)}

Fig. 4 shows variations in total monoterpene concentrations in c171 and c386 to the attack of Heterobasidion parviporum.

Kruskal-Wallis ANOVA between treatments within the same spruce clone, water regime and sampling time (Supplementary material Table S4) showed significant changes in total concentrations of monoterpenes (TCM) expressed as absolute amount ( $\mathrm{mg} \mathrm{g}^{-1}$ dry bark).

TCM were higher in all I tissues than in L samples or cortical tissues from $\mathrm{C}$ branches with only two exceptions: there were no differences in TCM between I tissues and L samples in D plants of c171 and c386 respectively 22 DAT and 44 DAT; these plants also showed a lower TCM in cortical tissues from $\mathrm{C}$ branches compared with samples from the disease treatments.

Sterile infection caused an increase in TCM in comparison with C branches in all seedlings of "relatively susceptible" c171, except W seedlings 22 DAT that showed a lower TCM when compared with the other treatments.

L samples in D seedlings of c386 44 DAT showed a higher TCM in comparison with C branches, while TCM decreased in L samples in D seedlings collected 22 DAT.

The Mann-Whitney $U$ Test within the same spruce clone, disease treatment and sampling time showed significant variations in TCM in response to drought stress (Table 4).

TCM in I tissues were lower in samples from D plants than in W plants of c171 and c386 collected, respectively, 22 DAT and 44 DAT.

Cortical tissues from C branches showed lower TCM in D plants than in W seedlings of c171 22 DAT, while higher TCM were observed in samples in D plants than in W plants of c386 and c171 22 DAT and 44 DAT, respectively.

Significant variations of TCM were also detected between the two clones within treatments and water plant status (Supplementary material Table S6).

Friedman Test within the same spruce clone, disease treatment and water status (Supplementary material Table S5) showed significant variations in TCM in response to sampling times.

I tissues in D plants of c171 showed lower TCM 22 DAT than 44 DAT, while higher TCM were observed in samples in W plants of c171 and in $\mathrm{W}$ and D plants of c386 22 DAT than 44 DAT.

I branches in W plants of $\mathrm{c} 171$ and D plants of c386 showed higher 


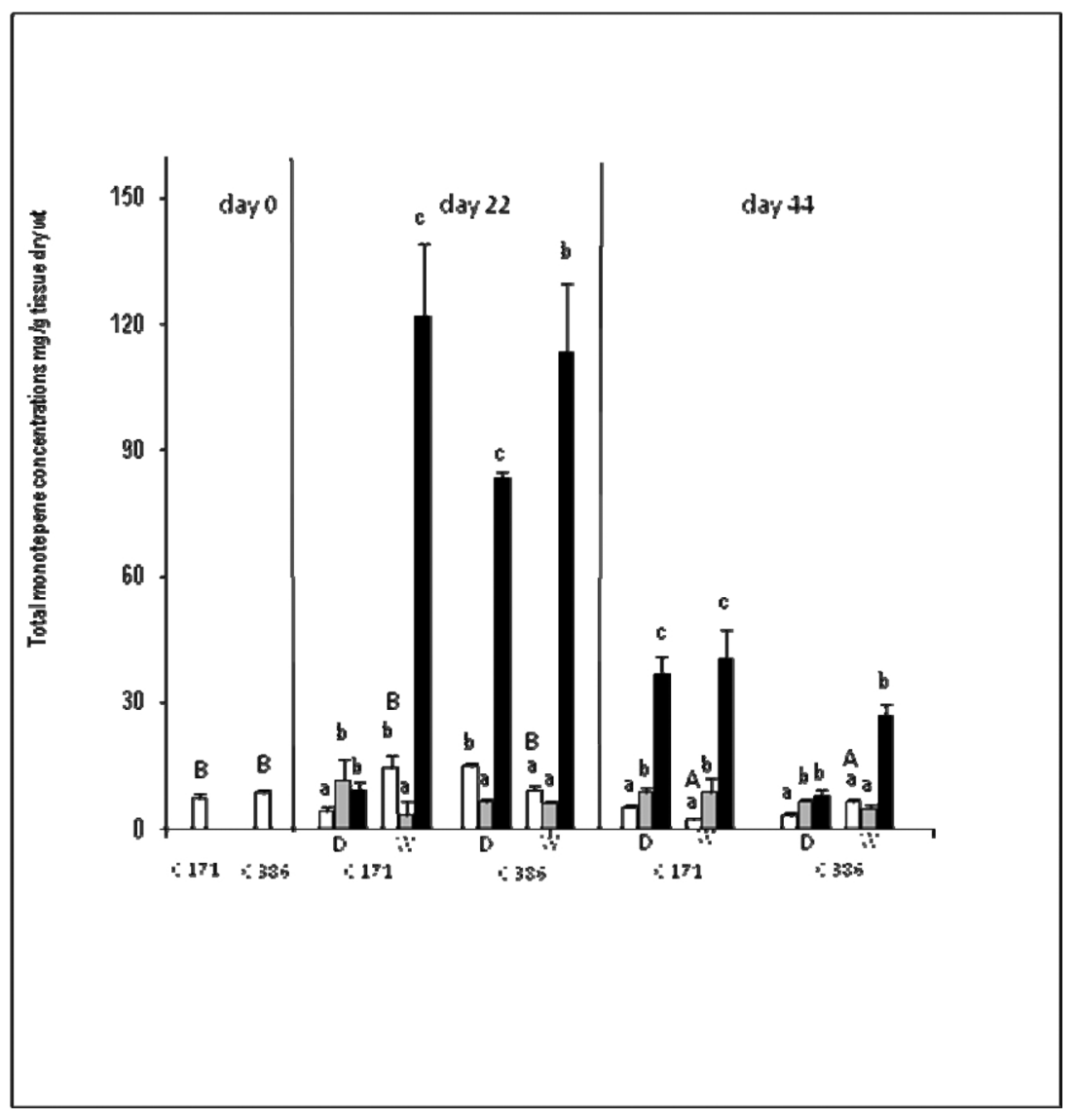

Fig. 4. Changes in total monoterpene concentrations (TCM) in the "relatively susceptible" clone 171 (c171) and the "relatively resistant" clone 386 (c386) to the attack of Heterobasidion parviporum. Treatments: drought-stressed plants (D); well-watered (W); cortical tissues from non-inoculated branches (C) (white bar); wounded plus inoculated with $H$. parviporum (black bar); wounded only (grey bar). Bars indicate means + standard errors. Different minor letters on the top of the bars indicate significantly different disease treatment means within the same clone, water regime and sampling time (Mann-Whitney $U$ test), while capital letters on the top of the bars indicate significant differences in TCM between different sampling times (day 0, day 22, day 44) in C samples within the same clone (Friedman test).

Table 4

Statistical results of the Mann-Whitney $U$ Test examining the variations of the total monoterpene concentrations in response to drought stress (d.f. $=1$ ) within Norway spruce clones, disease treatment and sampling time. Treatments: cortical tissues from non-inoculated branches (C); wounded plus inoculated with H. parviporum (I); wounded only (L); $p$-value where ${ }^{*} p<0.05$. NS: not significant. $\mathrm{N}^{\circ}$ samples: $3-5$ for each group.

\begin{tabular}{|c|c|c|c|c|c|c|c|}
\hline \multirow[t]{3}{*}{ Variable } & \multirow[t]{3}{*}{ Clone } & \multicolumn{6}{|l|}{$\chi^{2}(p)$} \\
\hline & & \multicolumn{2}{|l|}{ C } & \multicolumn{2}{|l|}{$\mathrm{L}$} & \multicolumn{2}{|l|}{ I } \\
\hline & & Day 22 & Day 44 & Day 22 & Day 44 & Day 22 & Day 44 \\
\hline \multirow[t]{2}{*}{ TCM } & c171 & $5.77^{*}$ & $4.81 *$ & $3.12 \mathrm{NS}$ & 0.54 NS & $5.33^{*}$ & $0.54 \mathrm{NS}$ \\
\hline & c386 & $4.25^{*}$ & $1.50 \mathrm{NS}$ & $0.20 \mathrm{NS}$ & $0.30 \mathrm{NS}$ & $1.84 \mathrm{NS}$ & $5.00 *$ \\
\hline
\end{tabular}

TCM 22 DAT than 44 DAT.

TCM in C branches in W plants of both clones showed a significant decrease at 44 DAT, while not significant changes in TCM were observed in samples of both clones between 0 DAT and 22 DAT.

\section{Discussion}

No significant differences in fungal growth were observed between the two clones. Fungal growth was reduced under drought stress treatment (D seedlings). At the end of the experiment, Heterobasidion colonization was increased in $\mathrm{W}$ seedlings. This result can be related with the intensity of stress and to non-monotonic relationships between water deficit and fungal infection, with an optimum at intermediate values. Lindberg and Johansson (1992) found that, in Norway spruce, resistance to fungal infection was elevated at high water potentials ( -0.3 to $-0.5 \mathrm{MPa})$ decreasing, at moderate drought stress $(-0.5$ to $-1.5 \mathrm{MPa})$ and increasing again at water potentials below $-1.5 \mathrm{MPa}$.
In the present experiment, trees were re-watered when predawn water potential was $-3.4 \mathrm{MPa}$ in "relatively susceptible" c171 and $-1.3 \mathrm{MPa}$ in c386. It is possible that the inoculated seedlings (D) grown in pots were probably quickly dehydrated when roots were subjected to drought stress, resulting in less carbon accessibility for fungal multiplication (Oliva et al., 2014). Available carbon reserves in W seedlings, conversely, may have facilitated the access of necrotrophic pathogens to essential carbon sources. On the other hand, long-term experiments are needed to clarify whether prolonged drought stress may reduce carbon reserves to an extent that limits the availability of carbohydrates to support defence, predisposing the xylem to attacks by fungal pathogens (La Porta et al., 2008).

As stress progressed, plants showed a reduction in predawn water potential, though with a larger drop in $\mathrm{c} 171$ (below - $3 \mathrm{MPa}$ ) than in c386, which can be related to differences in susceptibility to fungal infection between these Norway spruce clones. A marked decrease in water potential under drought conditions may cause an imbalance between carbon supply and demand, and the decrease in both carbon uptake (source) and growth (sink) in "relatively susceptible" clone may favour disease development, thus accelerating drought-induced tree mortality. Minimum predawn water potentials of about $-1.4 \mathrm{MPa}$ may already coincide with the threshold of water potential inducing significant xylem cavitation for this species (Cochard, 1992; Lu et al., 1996). At water potential below $-3 \mathrm{MPa}$, important reductions in xylem conductivity and stomatal conductance can be already detected (Borghetti et al., 1989; Mayr et al., 2003), and conductivity losses of up to $100 \%$ were found in Norway spruce with low water potential approaching - $4 \mathrm{MPa}$ (Mayr et al., 2002). The decrease in gas exchange preceded the decrease in water potential, reflecting an isohydric strategy (Lyr et al., 1992), reducing stomatal conductance at early stages of soil drought stress.

Reduced stomatal aperture in response to drought stress may have prevented Norway spruce seedlings from xylem dysfunction (Lu et al., 
1996). Because of the threshold-type relationship between stomatal conductance and net photosynthesis in conifers (Teskey et al., 1986), photosynthetic capacity may be sacrificed owing to reduced stomatal conductance. However, only $\mathrm{W}$ seedlings showed genotypic discrimination in gas exchange; these differences may be largely attributed to stomatal behaviour, since clone-related differences in predawn water potential were insignificant in $\mathrm{W}$ seedlings. Variation in intercellular $\mathrm{CO}_{2}$ concentration between treatments was unclear (data not shown) and a possible role of mesophyll metabolism in affecting leaf conductance of Norway spruce in long-term responses to drought stress cannot be ruled out. The relative reduction of stomatal conductance and net photosynthesis due to the decline in water availability reported here was comparable to that observed on maturing and mature Norway spruce in the field (Oren et al., 1986; Zimmermann et al., 1988; Lu et al., 1996; Ward et al., 2008). Nevertheless, differences between clones in stomatal conductance and net photosynthesis disappeared under water deficit conditions.

Patterns of net photosynthesis and stomatal conductance were similar, resulting in the close coupling of gas exchange components, without differences in instantaneous water use efficiency between clones (though c171 showed a delayed response of stomatal conductance and net photosynthesis to decreasing water potential in comparison with c386). Stomatal conductance and net photosynthesis were found to be strongly correlated in four-year-old seedlings of Norway spruce subjected to drought stress (Ditmarová et al., 2010). Pronounced sensitivity to drought stress is well known in Norway spruce, although not all physiological parameters display the same degree of sensitivity to dehydration, and may change in concert, enhancing the capability of trees to withstand water stress to some extent (Ditmarová et al., 2010).

The potential photochemical efficiency of PSII also decreased following drought stress, after an initial pronounced decline in gas exchange. Values reached at the peak of stress indicate impairment of photosynthetic machinery $\left(\mathrm{F}_{\mathrm{v}} / \mathrm{F}_{\mathrm{m}}<0.6\right.$ indicates sustained non-radiative energy dissipation), although this was reversible and promptly recovered to pre-stress values after re-watering. As in the case of water potential, chlorophyll fluorescence showed differences between clones in D plants, $\mathrm{c} 386$ being more tolerant than $\mathrm{c} 171$. Changes in chlorophyll fluorescence in young interior spruce trees during drought stress were found to reflect down-regulation of primary photochemistry and the augmentation of photoprotective mechanisms to avoid over-reduction and photoinhibitory damage (Eastman and Camm, 1995). A similar drop in the potential photochemical efficiency of PSII with drought stress was previously observed in Norway spruce (Pukacki and Kaminska-Rozek, 2005; Ditmarová et al., 2010); although, in contrast to our experiment, values did not recover to pre-stress conditions. Bigras (2005) observed an initial drop in the potential photochemical efficiency of PSII at leaf water potential ranging from -1 to $-2 \mathrm{MPa}$.

Marked differences in the constitutive monoterpene profiles were observed between the two clones; in particular, c386 showed high amounts of $\delta$-3-carene, $(-)$-limonene, $\gamma$-terpinene, and terpinolene, while c171 was characterized by high proportions of $(-)$ - $\alpha$-pinene, $(+)$ - $\alpha$-pinene, (-)- $\beta$-pinene, and (+)-limonene. Chemosystematic studies, mainly in conifer tree species, have demonstrated that, although the total concentrations of terpenes are clearly influenced by the environment, the constitutive monoterpene profiles in mature organs are stable and little affected by environmental factors; therefore, terpenes have proven to be very useful as biochemical markers in plant genetics to characterize species, provenances, hybrids and clones (Baradat et al., 1991; Hanover, 1992; Elzinga et al., 2015; Barbero and Maffei, 2016).

In Norway spruce, Kotzias et al. (1992) observed that monoterpene percentages including the enantiomers of $( \pm)$ - $\alpha$-pinene, remained constant throughout the year, while total concentrations of monoterpenes were strongly reduced during the summer months. In the present study, there were no differences in percentage monoterpene composition of cortical tissues from non-inoculated branches between drought-stressed and well-watered Norway spruce seedlings. Moreover, monoterpene profiles in cortical tissues from non-inoculated branches did not show significant variations among 0 DAT, 22 DAT and 44 DAT.

These findings support previous research showing that constitutive monoterpene profiles are not affected significantly by abiotic factors and remain stable (Langenheim, 1994; Tognetti et al., 1997; Michelozzi et al., 2004).

Previous experiments showed the discriminating power of terpene markers for studying clonal variation in Norway spruce (Boscherini and Michelozzi, 1993; Silvestrini et al., 2004). These variations in constitutive monoterpene profiles between different chemotype clones can have considerable consequences to plant chemical defence, since monoterpenes provide toxic, deterrent or inhibitory effects to herbivores, insects and microbial pathogens (reviewed by Gershenzon and Croteau, 1991; Langenheim, 1994). Therefore, differences in the constitutive monoterpene-rich resins between c171 and c386 may play an important role in the first line of the plant's defence mechanisms against infection with $H$. parviporum.

Conifer tree species have the potential to quantitatively vary monoterpenes in induced resins as we observed in the present experiment. Indeed, the infection generally caused some extensive compositional changes in monoterpenes. Sterile infection also affected relative concentrations of monoterpenes, but these reactions were less extensive than those elicited by the fungus. In particular, a higher proportion of $\delta$ 3 -carene was found constitutively in c386 in comparison with c171; moreover, infection with $H$. parviporum increased the relative contents of $\delta$-3-carene in all the samples, even if proportions of this monoterpene in I tissues of c386 were significantly higher than C branches only in $\mathrm{W}$ seedlings 44 DAT. $\delta$-3-Carene has been reported to be involved in some conifer-pest interactions (Krupa and Fries, 1971; Croteau et al., 1987; Pasquier-Barre et al., 2001; Fäldt et al., 2003) and also in the defense mechanism of spruce trees against the attack of Heterobasidion spp.

Forrest (1982) observed that $\delta$-3-carene, although present in small proportions relative to total monoterpene concentrations, was significantly higher in sitka spruce (Picea sitchensis) trees infected by H. annosum than uninfected plants. Woodward et al. (2007) found that in Sitka spruce clones less susceptible to $H$. annosum, the relative amount of $\delta$-3-carene was higher in the wounded plus inoculated and wounded only treatments compared to the samples collected $25 \mathrm{~cm}$ above the wound.

$\delta$-3-Carene also appears to be involved in the defensive chemistry of Norway spruce against the attack by $H$. parviporum; clones with a relatively high concentration of constitutive $\delta$-3-carene were more resistant to this pathogenic fungus than those with low concentrations (Michelozzi, data not published). In Norway spruce trees, the relative proportion of $\delta$-3-carene was found to increase in response to inoculation with $H$. parviporum (Zamponi et al., 2007).

The same expression pattern of changes in $\delta$-3-carene proportions was evident for terpinolene and these results were consistent with 3carene synthase that forms stereospecifically $(+)-3$-carene $(78 \%$ of total product) together with terpinolene ( $11 \%$ of total product) (Fäldt et al., 2003).

Also, the relative content of (-)-limonene increased significantly following fungal inoculation in all the samples. These data were not in agreement with previous results from Zamponi et al. (2007), who found that proportions of ( - )-limonene decreased significantly in 15-year-old Norway spruce trees grown in a field trial and infected by $H$. parviporum. Although this contrasting response could be due to differences in experimental design between studies, i.e., greenhouse pot vs field experiments, sampling time, and different ages of the plants, another possible explanation is that plant chemical defences can vary with plant ontogeny. Under the selective impacts of herbivores, trees during their juvenile-to-mature transition process can promote changes in terpene mixtures to reduce the incidence of pest and diseases (Hanover, 1992; Boege and Marquis, 2005).

In addition, some changes in the relative contents of $(-/+)-\alpha-$ 
pinene, myrcene, $(-/+)-\beta$-pinene, $p$-cymene and $\gamma$-terpinene, did not show a clear trend with disease treatments.

Although water stress induced significant variations in the proportions of several monoterpenes in the plants within the same spruce clone, disease treatment and sampling time, a similar trend in monoterpene responses to wounding plus inoculation and wounding only were observed in $\mathrm{W}$ and $\mathrm{D}$ plants.

No clear trend was observed in the proportions of several monoterpenes within the same spruce clone and disease treatment between different sampling times.

Finally, the results showing no significant changes in monoterpene profiles from $\mathrm{C}$ branches across 0 DAT, 22 DAT and 44 DAT, besides demonstrating the environmental stability of these compounds as mentioned above, strongly suggest that no systemic induction of monoterpenes occurred in Norway spruce seedlings.

Systemic induced resistance (SIR) is a form of defense mechanism that is induced in plant portions distal to localized infections and involving structural and biochemical responses. This mechanism occurs not only in herbaceous plants (Durrant and Dong, 2004), but also in different conifer tree species (Bonello et al., 2001; Bonello and Blodgett, 2003; Luchi et al., 2005; Wallis et al., 2007).

Although systemic induction of traumatic resin ducts was observed in Norway spruce (Krekling et al., 2004; Krokene et al., 2008), our data are in agreement with the study by Zhao et al. (2010) showing that terpene composition did not change in the untreated tissues after treatment and distinct chemical changes were restricted to the pretreated area of the stem.

It must be pointed out that terpenoid mixtures, either constitutive or induced, with their qualitative and quantitative compositional variations have more defensive values than single compounds. Additive or synergistic actions can enhance the ecological functions of different constituents composing the terpene mixture; moreover, the complexity of the terpenoids occurring in the mixtures, can impede the capacity of the herbivores to develop resistance to plant chemical defences (reviewed by Langenheim, 1994, Cates, 1996).

Therefore, certain combination of monoterpenes can confer greater protection than the content of individual components such as $\delta$-3carene, limonene, terpinolene, etc., against infection by $H$. parviporum in Norway spruce trees. Our results provide a foundation for future investigations on the relationships between terpene profiles and the attack of this fungus in order to characterize less susceptible Norway spruce chemotypes. Besides, a more comprehensive understanding of the function of antifungal properties of Norway spruce resin, requires knowledge of the defensive roles of sesquiterpenes and diterpenes and other compounds as phenols (Gershenzon and Dudareva, 2007).

An interesting observation of this study was the most copious production of monoterpenes occurring in I tissues. Differential elicitation of monoterpene synthesis is known in pest and pathogen attacks relative to mechanical wounds (Tomlin et al., 2000; Krekling et al., 2004; Woodward et al., 2007). Herbivore attacks yield more accumulation of defensive compounds in comparison with mechanically wounded trees (Cheniclet, 1987; Lieutier, 1993; Schmidt et al., 2005). The increase in monoterpene contents in inoculated tissues is due to defensive functions of these compounds that are dosage dependent (Gershenzon and Croteau, 1991; Langenheim, 1994; Cates, 1996).

Drought stress inhibited the production of monoterpenes in the induced resin of infected plants that did not result in enhanced susceptibility to $H$. parviporum. Indeed, fungal growth was lower in D than W seedlings. Although the majority of studies reported the contrary, some experiments showed that a specific abiotic stress may increase the resistance of plants to pests and diseases (reviewed by Suzuki et al., 2014).

It is worth noting to observe the decrease in TCM in cortical tissues from C branches in $\mathrm{W}$ plants of both clones 44DAT. A possible explanation is that the plant has already developed the capability to manage resource allocation and, therefore, the local need of resources to support defense production at the attack site can be met by translocation from distant sources within the individual tree.

\section{Conclusions}

In conclusion, the two clones showed different physiological response to drought stress, "relatively resistant" c386 being more tolerant than "relatively susceptible" c171. Overall, the range of physiological response of Norway spruce to different drought stress intensities indicated some degree of plasticity in carbon economy and water relations, though varying with the genotype. Differences in the relative content of constitutive monoterpenes and their enantiomers were observed between the two Norway spruce clones. Alterations in proportionate monoterpene composition and increase in total monoterpene concentrations occurred in response to the attack of $H$. parviporum. Monoterpene responses to mechanical wounds were also observed; however, these reactions were less extensive than responses to infection. In general, drought stress did not affect the monoterpene proportions in cortical tissues from non-inoculated branches. Although drought stress induced variations in the relative contents and decreases in the absolute amounts of monoterpenes, the trend in the relative proportions of monoterpenes in response to infection by $H$. parviporum was similar in $\mathrm{D}$ and $\mathrm{W}$ plants of both clones.

Our results on the defensive role of $\delta$-3-carene warrant further studies in order to see if this compound in combination with other monoterpenes may be used as biochemical markers for selection of less susceptible Norway spruce trees to infection by $H$. parviporum.

These findings provide a basis for future investigations on the relationships between terpene profiles and the attack by this fungus. A more comprehensive understanding of the function of antifungal properties of Norway spruce resin, requires knowledge of the defensive roles of the other terpene resin constituents such as sesquiterpenes and diterpenes, which can reinforce the biological activity of monoterpenes. A better knowledge of the ecological roles of terpenes may have implications for disturbance ecology and adaptive management of forests.

\section{Acknowledgments}

This work was supported by grants from the European Union Project QLRT-2000-00241 "Resistance of spruce to root and butt rot disease". Anat Madmony was supported by a fellowship of the Italian Ministry of Foreign Affairs. We thank Rodolfo Colom and Filippo Rossi for advice and help with ecophysiological measurements.

\section{Appendix A. Supplementary data}

Supplementary data associated with this article can be found, in the online version, at https://doi.org/10.1016/j.envexpbot.2018.03.007.

\section{References}

Allen, C.D., Macalady, A.K., Chenchouni, H., Bachelet, D., McDowell, N., Vennetier, M., Semerci, A., et al., 2010. A global overview of drought and heat-induced tree mortality reveals emerging climate change risks for forests. For. Ecol. Manage. 259, 660-684.

Arnerup, J., Lind, M., Olson, A., Stenlid, J., Elfstrand, M., 2011. The pathogenic white-rot fungus Heterobasidion parviporum triggers non-specific defence responses in the bark of Norway spruce. Tree Physiol. 31, 1262-1272.

Baradat, P., Marpeau, A., Walter, J., 1991. Terpene markers. In: Muller-Starck, G., Ziehe, M. (Eds.), Genetic Variation in European Populations of Forest Trees. Sauerlander's Verlag, Frankfurt am Main, pp. 40-66.

Barbero, F., Maffei, M., 2016. Biodiversity and chemotaxonomic significance of specialized metabolites. In: Arimura, G., Maffei, M. (Eds.), Plant Specialized Metabolism: Genomics. CRC Press, Taylor \& Francis, Boca Raton, pp. 23-66.

Barradas, C., Pinto, G., Correia, B., Castro, B.B., Phillips, A.J.L., Alves, A., 2018. Drought $\times$ disease interaction in Eucalyptus globulus under Neofusicoccum eucalyptorum infection. Plant Pathol. 67, 87-96.

Bigras, F.J., 2005. Photosynthetic response of white spruce families to drought stress. New For. 29, 135-148.

Blodgett, J.T., Kruger, E.L., Stansoz, G.R., 1997a. Sphaeropsis sapinea and water stress in a 
Red Pine plantation in Central Wisconsin. Ecol. Popul. Biol. 87, 429-434.

Blodgett, J.T., Kruger, E.L., Stansoz, G.R., 1997b. Effect of moderate water stress on disease development by Sphaeropsis sapinea on Red Pine. Ecol. Popul. Biol. 87, 422-428.

Bodles, W.J.A., Fossdal, C.G., Woodward, S., 2006. Multiplex real-time PCR detection of pathogen colonization in the bark and wood of Picea sitchensis clones differing in resistance to Heterobasidion annosum. Tree Physiol. 26, 775-782.

Boege, K., Marquis, R.J., 2005. Facing herbivory as you grow up: the ontogeny of resistance in plants. Trends Ecol. Evol. 20, 441-448.

Bonello, P., Blodgett, J.T., 2003. Pinus nigra-Sphaeropsis sapinea as a model pathosystem to investigate local and systemic effects of fungal infection of pines. Physiol. Mol. Plant Pathol. 63, 249-261.

Bonello, P., Gordon, T.R., Storer, A.J., 2001. Systemic induced resistance in Monterey pine. For. Pathol. 31, 99-106.

Borghetti, M., Raschi, A., Grace, J., 1989. Ultrasound emission after cycles of water stress in Picea abies. Tree Physiol. 5, 229-237.

Boscherini, G., Michelozzi, M., 1993. Capillary gas chomatography of the terpene components of Picea abies K. J. High Resolut. Chromatogr. 16, 619-620.

Brasier, C.M., Robredo, F., Ferraz, J.F.P., 1993. Evidence for Phytophthora cinnamomi involvement in Iberian oak decline. Plant Pathol. 42, 140-145.

Cates, R.G., 1996. The role of mixture and variation in the production of terpenoids in conifer-insect pathogen interactions. In: Romeo, J.T., Saunders, J.A., Barbosa, P. (Eds.), Phytochemical Diversity and Redundancy in Ecological Interactions. Plenum Press, New York, pp. 179-216.

Cheniclet, C., 1987. Effects of wounding and fungus inoculation on terpene producing systems of maritime pine. J. Exp. Bot. 38, 1557-1572.

Cobb, F.W., Krstic, M., Zavarin, E., Barber Jr., H.W., 1968. Inhibitory effects of volatile oleoresin components on Fomes annosus and four Ceratocystis species. Phytopathology $58,1327-1335$.

Cochard, H., 1992. Vulnerability of several conifers to air embolism. Tree Physiol. 11, 73-83.

Croisé, L., Lieurier, F., Cochard, H., Dreyer, E., 2001. Effect of drought stress and high density stem inoculation with Leptographium wingfieldii on hydraulic properties of young Scots pine trees. Tree Physiol. 21, 427-436.

Croteau, R., Gurkewitz, S., Johnson, M.A., Fisk, H.J., 1987. Biochemistry of oleoresinosis: monoterpene and diterpene biosynthesis in lodgepole pine saplings infected with Ceratocystis clavigera or treated with carbohydrate elicitors. Plant Physiol. 85, 1123-1128.

Das, T., Hajong, M., Majumdar, D., Tombisana Devi, R.K., Rajesh, T., 2016. Climate change impacts on plant diseases. SAARC J. Agric. 14, 200-209.

Desprez-Loustau, M.L., Marçais, B., Nageleisen, L.M., Piou, D., Vannini, A., 2006. Interactive effects of drought and pathogens in forest trees. Ann. For. Sci. 63, 597-612.

Dimitri, L., 1994. Host defence and genetical resistance of Norway spruce (Picea abies (L.) Karst.) against Heterobasidion annosum. In: Stenlid, J. (Ed.), Proc. Eight Int. Conf. on Root and Butt Rots. Wik, Sweden and Haikko Finland, pp. 1-9.

Ditmarová, L., Kurjak, D., Palmroth, S., Kmet', J., Střelcová, K., 2010. Physiological responses of Norway spruce (Picea abies) seedlings to drought stress. Tree Physiol. 30, 205-213.

Durrant, W.E., Dong, X., 2004. Systemic acquired resistance. Annu. Rev. Phytopathol. 42, $185-209$.

Eastman, P.A.K., Camm, E.L., 1995. Regulation of photosynthesis in interior spruce during water stress: changes in gas exchange and chlorophyll fluorescence. Tree Physiol. 15, 229-235.

Elzinga, S., Fischedick, J., Podkolinski, R., Raber, J.C., 2015. Cannabinoids and terpenes as chemotaxonomic markers in cannabis. Nat. Prod. Chem. Res. 3, 4.

Fäldt, J.F., Martin, D., Miller, B., Rawat, S., Bohlmann, J., 2003. Traumatic resin defense in Norway spruce (Picea abies): methyl jasmonate-induced terpene synthase gene expression, and cDNA cloning and functional characterization of $(+)$-3-carene synthase. Plant Mol. Biol. 51, 119-133.

Forrest, G.I., 1982. Preliminary work on the relation between resistance to Fomes annosus and the monoterpene composition of Sitka spruce resin. In: Heybroek, H.M., Stephan, B.R., von Weissenberg, K. (Eds.), Resistance to Diseases and Pests in Forest Trees. PUDOC, Wageningen, pp. 194-197.

Gaylord, M.L., Kolb, T.E., Pockman, W.T., Plaut, J.A., Yepez, E.A., Macalady, A.K. Pangle, R.E., McDowell, N.G., 2013. Drought predisposes piñon-juniper woodlands to insect attacks and mortality. New Phytol. 198, 567-578.

Gershenzon, J., Croteau, R., 1991. Terpenoids. In: Rosenthal, G.A., Berenbaum, M.R. (Eds.), Herbivores, Their Interactions with Secondary Metabolites. Academic Press, New York, pp. 169-219.

Gershenzon, J., Dudareva, N., 2007. The function of terpene natural products in the natural world. Nat. Chem. Biol. 3, 408-414.

Gori, Y., Cherubini, P., Camin, F., La Porta, N., 2013. Fungal root pathogen (Heterobasidion parviporum) increases drought stress in Norway spruce stand at low elevation in the Alps. Eur. J. For. Res. 132, 607-619.

Hammerschmidt, R., 1999. Induced disease resistance: how do induced plants stop pathogens? Physiol. Mol. Plant Pathol. 55, 77-84.

Hanewinkel, M., Cullmann, D.A., Schelhaas, M.J., Nabuurs, G.-J., Zimmermann, N.E., 2013. Climate change may cause severe loss in the economic value of European forest land. Nat. Clim. Change 3, 203-207.

Hanover, J.W., 1992. Applications of terpene analysis in forest genetics. New For. 6, 159-178.

Hietala, A.M., Eikenes, M., Kvaalen, H., Solheim, H., Fossdal, C.G., 2003. Multiplex realtime PCR for monitoring. Appl. Environ. Microbiol. 69, 4413-4420.

Karlsson, P.E., Medin, E.L., Wallin, G., Sellden, G., Skarby, L., 1997. Effects of ozone and drought stress on the physiology and growth of two clones of Norway spruce (Picea abies). New Phytol. 136, 265-275.

Korhonen, K., Stenlid, J., 1998. Biology of Heterobasidion annosum. In: Woodward, S. Stenlid, J., Karjalainen, R., Hüttermann, A. (Eds.), Heterobasidion Annosum: Biology, Ecology, Impact and Control. CAB, pp. 43-70.

Korhonen, K., 1978. Intersterility groups of Heterobasidion annosum. Commun. Inst. For. Fenniae 94, 1-25.

Kotzias, D., Sparta, C., Duane, C., 1992. Distribution of optical isomers of monoterpenes ( \pm )- $\alpha$-pinene in the leaf oil of conifers. Naturwissenschafien 92, 24-26.

Krekling, T., Franceschi, V.R., Krokene, P., Solheim, H., 2004. Differential anatomical response of Norway spruce stem tissues to sterile and fungus infected inoculations. Trees 18, 1-9.

Krokene, P., Nagy, N.E., Solheim, H., 2008. Methyljasmonate and oxalicacid treatment of Norway spruce: anatomically based defense responses and increate resistance against fungal infection. Tree Physiol. 28, 29-35.

Krupa, S., Fries, N., 1971. Studies on ectomycorrhizae of pine: I. Production of volatile organic compounds. Can. J. Bot. 49, 1425-1431.

La Porta, N., Capretti, P., Thomsen, I.M., Kasanen, R., Hietala, A.M., Von Weissenberg, K., 2008. Forest pathogens with higher damage potential due to climate change in Europe. Can. J. Plant. Pathol. 30, 177-195.

Langenheim, J.H., 1994. Higher plant terpenoids: a phytocentric overview of their ecological roles. J. Chem. Ecol. 20, 1223-1280.

Lieutier, F., 1993. Induced defense reactions of conifers to bark beetles and their associated Ophiostoma. In: Wingfield, M.J., Seifert, K.A., Webber, J.F. (Eds.), Ceratocystis and Ophiostoma: Taxonomy, Biology and Pathogenicity. American Phytopathology Society Press, St. Paul, M.N, pp. 206-215.

Linares, C.J., Camarero, J.J., Bowker, M.A., et al., 2010. Stand-structural effects on Heterobasidion abietinum-related mortality following drought events in Abies pinsapo. Oecologia 164, 1107-1119.

Lindberg, M., Johansson, M., 1989. Resistance of Norway spruce seedlings to infection by Heterobasidion annosum in relation to drought stress. In: Morrison, D. (Ed.), Proc. Seventh Int. Conf. on Root and Butt Rots. Vernon and Victoria, British Columbia, Canada, pp. 615-622.

Lindberg, M., Johansson, M., 1992. Resistance of Picea abies seedlings to infection by Heterobasidion annosum in relation to drought stress. Eur. J. For. Pathol. 22, 115-124.

Lu, P., Biron, P., Granier, A., Cochard, H., 1996. Water relations of adult Norway spruce (Picea abies (L.) Karst) under soil drought in the Vosges mountains: whole-tree hydraulic conductance, xylem embolism and water loss regulation. Ann. For. Sci. 53, $113-121$.

Luchi, N., Ma, R., Capretti, P., Bonello, P., 2005. Systematic induction of traumatic resin ducts and resin flow in Austrian pine by wounding and inoculation with Sphaeropsis sapinea and Diplodia scrobiculata. Planta 221, 75-84.

Lyr, H., Fiedler, H.J., Tranquilini, W., 1992. Physiologie und Ökologie der Gehölze. G. Fischer Verlag, Stuttgart.

Madar, Z.Z., Solel, Z., Kimchi, M., 1989. Effect of water stress in cypress on the development of cancers caused by Diplodia pinea f. sp. cupressi and Seiridium cardinale. Plant Dis. 73, 484-486.

Madar, Z., Solel, Z., Riov, J., Sztejnberg, A., 1995. Phytoalexin production by cypress in response to infection by Diplodia pinea f. sp. cupressi and its relation to water stress. Physiol. Mol. Plant Pathol. 47, 29-38.

Madar, Z., Kimchi, M., Solel, S., 1996. Fusarium canker of Italian cypress. Eur. J. For. Pathol. 26, 107-112.

Mayr, S., Wolfschwenger, M., Bauer, H., 2002. Winter-drought induced embolism in Norway spruce (Picea abies) at the Alpine timberline. Physiol. Plant. 115, 74-80.

Mayr, S., Schwienbacher, F., Bauer, H., 2003. Winter at the Alpine Timberline. Why does embolism occur in Norway spruce but not in stone pine? Plant Physiol. 131, 780-792.

McDowell, N.G., Beerling, D.J., Breshears, D.D., Fisher, R.A., Raffa, K.F., Stitt, M., 2011. The interdependence of mechanisms underlying climate-driven vegetation mortality. Trends Ecol. Evol. 26, 523-532.

Michelozzi, M., Tognetti, R., Maggino, F., Radicati, M., 2004. Seasonal variations in monoterpene profiles and ecophysiological traits in Mediterranean pine species of group halepensis. iForest 1, 65-74.

Modrzynski, J., Erikkson, G., 2002. Response of Picea abies populations from elevational transects in the Polish Sudety and Carpathia mountains to simulated drought stress. For. Ecol. Manage. 165, 105-116.

Niemela, T., Korhonen, K., 1998. Taxonomy of the genus Heterobasidion. In: Woodward, S., Stenlid, J., Karjalainen, R., Huttermann, A. (Eds.), Heterobasidion annosum Biology, Ecology, Impact and Control. CAB International, Wallingford, pp. 27-34.

Niinemets, Ü., 2010. Responses of forest trees to single and multiple environmental stresses from seedlings to mature plants: past stress history, stress interactions, tolerance and acclimation. For. Ecol. Manage. 260, 1623-1639.

Oliva, J., Thor, M., Stenlid, J., 2010. Reaction zone and periodic increment decrease in Picea abies trees infected by Heterobasidion annosum s.1. For. Ecol. Manage. 260, 692-698.

Oliva, J., Camarero, J.J., Stenlid, J., 2012. Understanding the role of sapwood loss and reaction zone formation on radial growth of Norway spruce (Picea abies) trees decayed by Heterobasidion annosum s.l. For. Ecol. Manage. 274, 201-209.

Oliva, J., Stenlid, J., Martínez-Vilalta, J., 2014. The effect of fungal pathogens on the water and carbon economy of trees: implications for drought-induced mortality. New Phytol. 203, 1028-1035.

Oren, R., Schulze, E.D., Matyssek, R., Zimmermann, R., 1986. Estimating photosynthetic rate and annual carbon gain in conifers from specific leaf weight and leaf biomass. Oecologia 70, 187-193.

Pasquier-Barre, F., Palasse, C., Goussard, F., Auger-Rozenberg, M.A., Géri, C., 2001. Relationship of scots pine clone characteristics and water stress to hatching and larval performance of the sawfly Diprion pini (Hymenoptera: Diprionidae). Environ. Entomol. 30, 1-6. 
Phillips, M.A., Croteau, R.B., 1999. Resin-based defenses in conifers. Trends Plant Sci. 4, $184-190$

Puddu, A., Luisi, N., Capretti, P., Santini, A., 2003. Environmental factors related to damage by Heterobasidion abietinum in Abies alba forests in Southern Italy. For. Ecol. Manage. 180, 37-44.

Pukacki, P.M., Kaminska-Rozek, E., 2005. Effect of drought stress on chlorophyll a fluorescence and electrical admittance of shoots in Norway spruce seedlings. Trees 19, 539-544.

Sancho-Knapik, D., Angeles Sanz, M., Peguero-Pina, J.J., Niinemetes, U., Gil-Pelegrini, E., 2017. Changes of secondary metabolites in Pinus sylvestris L. needles under increasing soil water deficit. Ann. For. Sci. 74, 24-34.

Schmidt, A., Zeneli, G., Hietala, A.M., Fossdal, C.G., Krokene, P., Christiansen, E., Gershenzon, J., 2005. Induced chemical defenses in conifers: biochemical and molecular approaches to studying their function. In: Romeo, J.T. (Ed.), Chemical Ecology and Phytochemistry in Forest Ecosystems. Elsevier, Amsterdam, pp. 1-28.

Schreiber, U., Bilger, W., Neubauer, C., 1994. Chlorophyll fluorescence as a nonintrusive indicator for rapid assessment of in vivo photosynthesis. Ecophysiology of Photosynthesis. Springer, Berlin, pp. 49-70.

Seidl, R., Schelhaas, M.J., Lexer, M.J., 2011. Unraveling the drivers of intensifying forest disturbance regimes in Europe. Glob. Change Biol. 17, 2842-2852.

Silvestrini, E., Michelozzi, M., Skroppa, T., Brancaleoni, E., Ciccioli, P., 2004. Characterisation of different clones of Picea abies (L.) Karst using head-space sampling of cortical tissues combined with enantioselective capillary gas chromatography for the separation of chiral and nonchiral monoterpenes. J. Chromatogr. A 1034, 183-189.

Suzuki, N., Rivero, R.M., Shulaev, V., Blumwald, E., Mittler, R., 2014. Abiotic and biotic stress combinations. New Phytol. 203, 32-43.

Swedjemark, G., Stenlid, J., 1994. Variation among Norway spruce clones and Heterobasidion annosum isolates in greenhouse inoculations. In: Stenlid, J. (Ed.), Proc. Eight Int. Conf. on Root and Butt Rots. Wik, Sweden and Haikko Finland, pp. 10-16.

Swedjemark, G., Stenlid, J., Karlsson, B., 1997. Genetic variation among clones of Picea abies in resistance to growth of Heterobasidion annosum. Silvae Genetica 46, 369-374.

Swedjemark, G., Stenlid, J., Karlsson, B., 2001. Variation in growth of Heterobasidion annosum among clones of Picea abies incubated for different periods of time. For. Pathol. 31, 163-175.

Teskey, R.O., Fites, J.A., Samuelson, L.J., Bongarten, B.C., 1986. Stomatal and non stomatal limitations to net photosynthesis in Pinus taeda L. under different environmental conditions. Tree Physiol. 2, 131-142.

Tognetti, R., Michelozzi, M., Giovannelli, A., 1997. Geographical variations in water relations, hydraulic architecture and terpene composition in Aleppo pine seedlings from Italian provenances. Tree Physiol. 17, 241-250.

Tomlin, E.S., Antonejevic, E., Alfaro, R.I., Borden, J.H., 2000. Changes in volatile terpene and diterpene resin acid composition of resistant and susceptible white spruce leaders exposed to simulated white pine weevil damage. Tree Physiol. 20, 1087-1095.

Ununger, J., Ekberg, I., Hyun, K., 1988. Causal relations between juvenile shoot growth characters in Picea abies. Scand. J. For. Res. 3, 147-156.

Wallis, C., Eyles, A., Chorbadjian, R., McSpadden Gardener, B., Hansen, R., Cipollini, D., Herms, D.A., Bonello, P., 2007. Systemic induction of phloem secondary metabolism and its relationship to resistance to a canker pathogen in Austrian pine. New Phytol. 177, 767-778.

Ward, E.J., Oren, R., Sigurdsson, B.D., Jarvis, P.G., Linder, S., 2008. Fertilization effects on mean stomatal conductance are mediated through changes in the hydraulic attributes of mature Norway spruce trees. Tree Physiol. 28, 579-596.

Wargo, P.M., Harrington, T.C., 1991. Host stress and susceptibility. In: In: Shaw, C.G., Kile, G.A. (Eds.), Armillaria Root Disease, vol. 691. USDA Hand book, pp. 88-101.

Wong, C.M., Daniel, L.D., 2017. Novel forest decline triggered by multiple interactions among climate, an introduced pathogen and bark beetles. Glob. Change Biol. 23, 1926-1941.

Woodward, S., Stenlid, J., Karjalainen, R., Hutterman, A., 1998. Heterobasidion annosum: Biology, Ecology, Impact and Control. CAB pp. 589.

Woodward, S., Bianchi, S., Bodles, W.J., Beckett, L., Michelozzi, M., 2007. Physical and chemical responses of Sitka spruce (Picea sitchensis) clones to colonization by Heterobasidion annosum as potential markers for relative host susceptibility. Tree Physiol. 27, 1701-1710.

Zamponi, L., Michelozzi, M., Capretti, P., 2007. Terpene response of Picea abies and Abies alba to infection with Heterobasidion s.1. For. Pathol. 37, 243-250.

Zhao, T., Krokene, P., Björklund, N., Långström, B., Solheim, H., Christiansen, E., BorgKarlson, A.K., 2010. The influence of Ceratocystis polonica inoculation and methyl jasmonate application on terpene chemistry of Norway spruce, Picea abies. Phytochemistry 71, 1332-1341.

Zimmermann, R., Oren, R., Schulze, E.D., Werk, K.S., 1988. Performance of two Pice abies (L.) Karst. stands at different stages of decline. II. Photosynthesis and leaf conductance. Oecologia 76, 513-518. 